\title{
Caracterización granulométrica de los depósitos de abanicos aluviales en la Cuenca de Motozintla, Chiapas, México: un peligro geológico latente por eventos de inundación
}

Juan M. Sánchez-Núñez, José Luis Macías, Ricardo Saucedo, David A. Novelo, José J. Zamorano, Fabiola Mendiola

Juan M. Sánchez-Núñez sanchez0120@gmail.com

Instituto Politécnico Nacional-CIIEMAD. Calle 30 de junio de $1520 \mathrm{~s} / \mathrm{n}$, Barrio La Laguna Ticomán, Del. Gustavo A. Madero, C.P. 07340, CDMX

\section{José Luis Macías}

Fabiola Mendiola

Instituto de Geofísica, UNAM Campus Morelia. Antigua carretera a Pátzcuaro no. 8701 , Col. Ex-Hacienda San José de la Huerta C.P. 58190, Morelia, Michoacán, México.

\section{Ricardo Saucedo}

Instituto de Geología, UASLP. Dr. Manuel Nava no. 5, Zona Universitaria C.P. 78240, San Luis Potosí, SLP, México.

\section{David A. Novelo}

Departamento de Sismología, Instituto de Geofísica, UNAM, Delegación Coyoacán, CDMX.

\section{José J. Zamorano}

Departamento de Geografía Física, Instituto de Geografía, UNAM, Delegación Coyoacán, CDMX

BOL. SOC. GEOL. MEX. 2017

VOL. 69 NO. 3

P. $529-554$

Manuscrito recibido: Marzo 10, 2015. Manuscrito corregido: Abril 18, 2017. Manuscrito aceptado: Abril 27, 2017.

\section{RESUMEN}

La cuenca de Motozintla se localiza en la región montañosa del sur del estado de Chiapas, México, donde los flujos de escombros han sido eventos frecuentes. Estos fenómenos naturales representan un peligro geológico para los habitantes por ser súbitos, por su alta energía, asociados a pendientes inestables $\left(>30^{\circ}\right)$, topografía abrupta y factores antropogénicos que hacen más propensa a la población a sufrir los impactos de estos flujos. Esta cuenca se localiza en el extremo occidental de la falla lateral izquierda Polochic, que separa las placas de Norte América y El Caribe. Por su ubicación tectónica, esta cuenca está formada por abanicos aluviales y terrazas colgadas en ambas partes del límite tectónico. Este trabajo se enfoca al estudio de los abanicos aluviales, que están formados por sucesiones de depósitos, algunos separados por paleosuelos poco desarrollados. Se determinaron las características granulométricas totales de 57 depósitos muestreados en 30 columnas estratigráficas. Estas características indican que los abanicos aluviales están compuestos en su mayoría por flujos de escombros (polimíctico y muy pobremente seleccionado) inmaduros, transportados en pendientes abruptas ( $>$ $30^{\circ}$ ) y con distancias de transporte inferiores a $10 \mathrm{~km}$ desde la fuente de origen. Estos resultados no se habían presentado en México para este tipo de ambientes tectónicos y de depósito. La recurrencia de estos eventos en el pasado indica que han ocurrido flujos de escombros similares causados por fenómenos hidrometerológicos y sísmicos.

Palabras clave: Motozintla, abanicos aluviales, flujos de escombros, peligro geológico.

\section{ABSTRACT}

Motozintla basin is situated in the southern mountainous region of Chiapas State, Mexico, where debris flows have frequently occurred. These natural phenomena are latent geohazards for the population of $\mathrm{Mo}^{-}$ tozintla. Their sudden nature, their high energy, related unstable slopes $\left(>30^{\circ}\right)$, rugged topography and anthropogenic footprint contribute to make them more dangerous. This basin is located in the western end of the left lateral Polochic fault - the structural limit of North America and the Caribbean plates. Tectonically, the basin encompasses alluvial fans and terraces that cut both sides of the plate boundary. This study deals with the alluvial fans that are composed of successive deposits, some separated by underdeveloped paleosoils. Granulometric characteristics of 57 deposits sampled in 30 stratigraphic columns were determined. The results revealed that most alluvial fans were sourced from immature flows (lithological, heterometric and various other sources) transported on steep slopes $\left(>30^{\circ}\right)$ with less than $10 \mathrm{~km}$ of transport from the source. The repeated occurrence of these events in the past indicates that the events of debris flow are influenced by hydrometerological and seismological processes. This research is the first of its kind in an active tectonic setting of Mexico.

Keywords: Motozintla, alluvial fans, debris flows, geohazard. 


\section{Introducción}

En septiembre de 1998 y octubre de 2005 los municipios de Motozintla y Tapachula, Chiapas sufrieron los desastres históricos más significativos registrados hasta la fecha (Caballero et al., 2006; Murcia y Macías, 2009). Lluvias intensas provocadas por la tormenta tropical "Earl" en el Atlántico y el huracán "Stan" en el Pacífico, respectivamente, causaron diversos procesos de remoción en masa, principalmente del tipo de flujos de escombros. La zona más dañada por ambos eventos fue el Municipio de Motozintla localizado en el fondo del valle en forma de "V", desarrollado justo en la confluencia de los ríos Xelajú Grande, La Mina y Allende.

Los desastres hidrometeorológicos de 1998 y 2005 han demostrado que Motozintla se encuentra en una zona poco segura, vulnerable a inundaciones y remoción de materiales por corrientes hídricas y por procesos asociados a la gravedad. Recientemente, los desastres por procesos naturales han incrementado su potencial de daño debido a la intensa actividad antrópica como la deforestación, el pastoreo de animales domésticos y la construcción de carreteras; estas actividades causan la inestabilidad en las laderas (Caballero et al., 2006; Sánchez-Núñez et al., 2012). Las condiciones geológicas y climáticas en Motozintla, Chiapas han provocado el emplazamiento de grandes volúmenes de detritos sobre la planicie aluvial del río Xelajú Grande durante los últimos 25000 años (Sánchez-Núñez et al., 2015). Estos depósitos y su registro estratigráfico obtenido en abanicos aluviales y terrazas han permitido identificar eventos históricos, provocados por fenómenos meteorológicos y sísmicos tan jóvenes como 165 años (Sánchez-Núñez et al., 2015). La cuenca de Motozintla, localizada en el sureste de México (Figura 1A), tiene ejemplos de depósitos emplazados por fenómenos hidrometeorológicos ocurridos en 1998 y 2005, que dan cuenta de su potencial para afectar vidas humanas y destruir sus propiedades. En este estudio se describen los parámetros granulométricos de los depósitos que forman los abanicos aluviales para conocer la distribución de clastos en sus facies proximales y medias, su morfología y su correlación con las fuentes de aporte. Estas características podrían ser utilizadas en ambientes similares en otras regiones del país y del mundo.

\section{Marco tectónico y geología local}

La cuenca de Motozintla se localiza en una zona tectónica compleja que involucra el límite tectónico lateral entre las placas Norteamericana y del Caribe, las cuales son subducidas por la placa de Cocos, dando origen a la Unión Triple de Tehuantepec (Espíndola, 1996). Esta triple unión se considera inestable y muy compleja, ya que en los últimos ocho millones de años su posición ha migrado a lo largo de la falla Polochic (Figura 1B). Guzmán-Speziale (2010) concluyó que en su porción oeste las placas Norteamericana y Caribe están representadas por fallas laterales izquierdas, con orientación general $\mathrm{E}-\mathrm{W}$ y de alta sismicidad. La sismicidad ha sido evidenciada por la ocurrencia de sismos de hasta $\mathrm{M}=7.6$ asociados a la falla Concordia, situada al norte del sistema de fallas Polochic en el sureste de México. Anderson et al. (1973) describieron las fallas Chixoy-Polochic y Motagua con dirección E-W que cruzan Guatemala y Chiapas (Figura 1B). Muehlberger y Ritchie (1975) también describieron esta zona de fallas como Cuilco-Chixoy-Polochic y describieron que se internaban al oeste en México. El rasgo más significativo de esta interpretación es la bifurcación de la falla Polochic en la región de Amatenango de la Frontera del municipio de Motozintla de Mendoza, la cual presenta una actitud estructural hacia el N $60^{\circ}$ E. Posteriormente, Burkart (1978) concluyó que la Falla Polochic tenía una componente lateral izquierda con una longitud aproximada de $350 \mathrm{~km}$ desde la Falla Motagua hasta la trinchera Mesoamericana.

Burkart (1983) consideró que las rocas en ambos lados de la falla han sufrido un desplazamiento lateral de $132 \mathrm{~km}$. Por lo tanto, existe un contraste litológico entre la zona norte de la falla Polochic, 


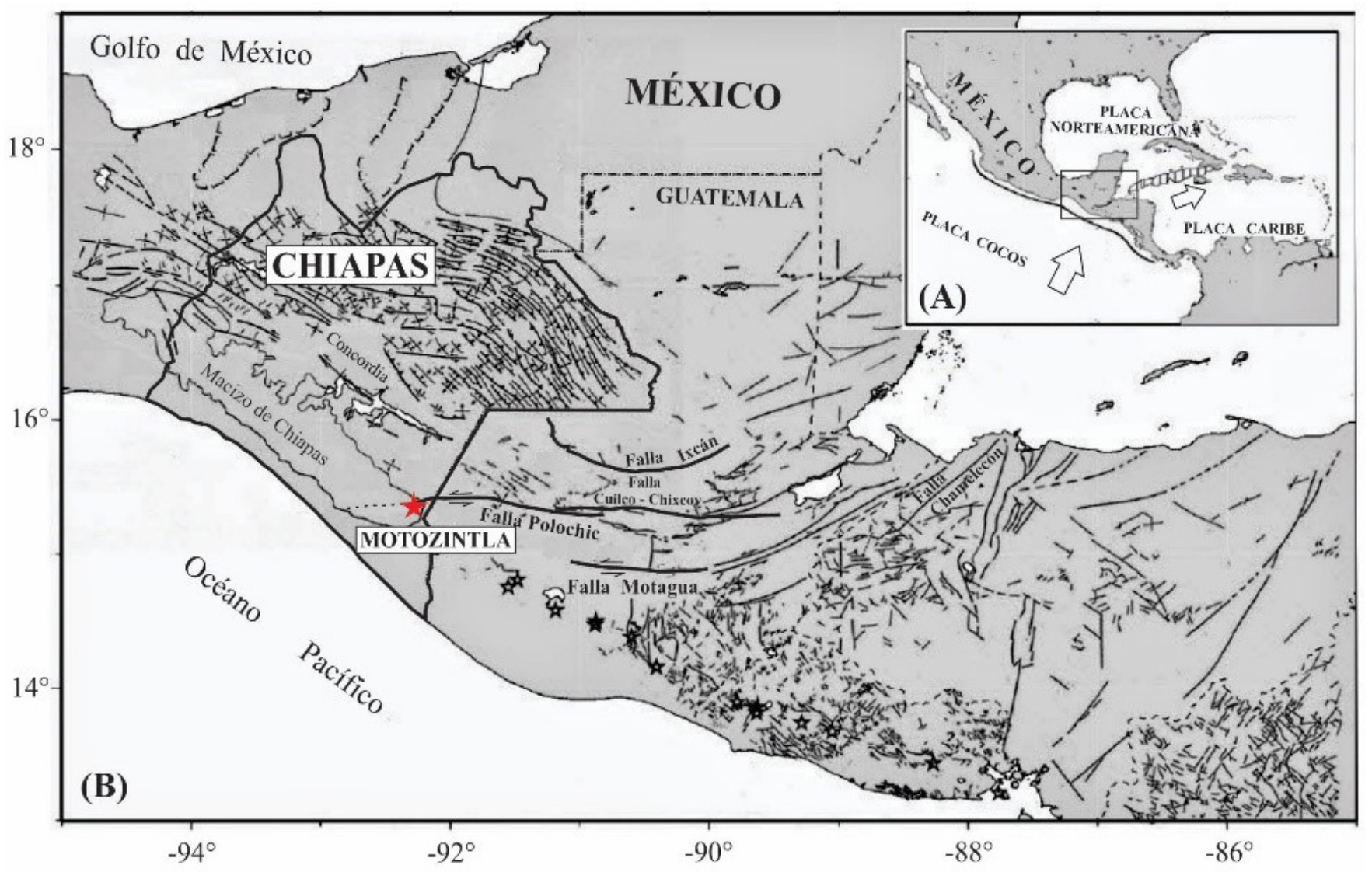

Figura 1 (A) Localización geográfica del área de estudio y su relación con las placas tectónicas de Norteamérica, Caribe y Cocos. (B) Marco tectónico de la región de Motozintla y su localización sobre la traza del sistema de fallas Polochic-Motagua. Modificada de Guzmán-Speziale (2010).

ya que las rocas del Paleozoico Tardío y Mesozoico se encuentran menos deformadas que las rocas cristalinas de la zona sur. Este desplazamiento de $130 \mathrm{~km}$, también coincide con el modelo presentado por Authemayou et al. (2011) en el que define a la triple unión como la interacción de las placas Norte América-Cocos-Caribe en un arreglo subducción-subducción-transformante (SST). Molina-Garza et al. (2015) también proponen que la falla Tonalá-Motozintla constituye la sutura que forma esta triple unión y que tiene una migración hacia el sur, asumiendo que presenta una componente de empuje cercano a la intersección con el sistema de fallas Polochic.

Lyon-Caen et al. (2006) y Franco et al. (2012) realizaron estudios detallados sobre la cinemática de fallas en el norte de América Central y su conexión con la zona de subducción de la placa de Cocos en la región de Chiapas, Guatemala y El Salvador. El estudio evidenció el predominio del movimiento lateral y la velocidad de movimiento (vía GPS) de las estructuras volcano-tectónicas mayores. Además, permitió elaborar modelos cinemáticos críticos para la región de la triple unión (Norteamericana/Cocos/Caribe), en particular para la región de Chiapas y la zona de subducción del sur de México.

Guzmán-Speziale y Meneses-Rocha (2000) propusieron una "Provincia de fallas de rumbo", la cual cubre la mayor parte de la Sierra de Chiapas. La característica principal de esta provincia es la alternancia de horsts y grabens, limitados por fallas la- 
terales izquierdas, predominando una orientación E-W y un arreglo en echelon en los bloques levantados; en los grabens, las fallas a rumbo se vuelven divergentes (Caballero et al., 2006; Salazar, 2008). Localmente, Caballero et al. (2006) concluyeron que la región de Motozintla está sujeta a un esfuerzo de compresión, evidenciado por fallas inversas con orientación NW-SE expuestas al norte del río Xelajú Grande y fallas normales con orientación $\mathrm{NE}-\mathrm{SW}$ al sur de este río. Ambos sistemas de fallas generan facetas triangulares y abanicos aluviales truncados sobre la planicie aluvial de dicho río. En lo referente a la estratigrafía local, la unidad más antigua (basamento) está constituida por rocas metamórficas de bajo grado del Precámbrico, esquistos de anfibolita-moscovita y gneis de biotita-anfibolita que se distribuyen de forma irregular como remanentes o colgantes (roof pendants) en el Macizo de Chiapas (Mérida, 1976; Carfantan, 1977; Caballero et al., 2006).

La roca con mayor distribución en el área de estudio $(\sim 60 \%)$ es el Macizo de Chiapas de edad Pérmico Tardío-Triásico Temprano (265-224 Ma); esta roca constituye el mayor cuerpo intrusivo del estado de Chiapas y está formado por un granito de biotita color rosa ligeramente bandeado (Múgica, 1987). Lo anterior concuerda con Weber et al. (2002), quien menciona que la Sierra de Chiapas está compuesta en su mayor parte por rocas ígneas del batolito chiapaneco y que representa uno de los complejos ígneos más grandes de México.

Molina-Garza et al. (2015) concluyeron que el macizo de Chiapas está constituido por un complejo plutónico y metamórfico de edad predominantemente Pérmico, sobre el que yacen rocas sedimentarias de edad Jurásico-Cretácico. También mencionan que el plutón de la costa chiapaneca está sobreyacido por una sucesión Jurásica de lechos rojos (red beds). Al norte de la cuenca de Motozintla afloran lechos rojos de la Formación Todos Santos sobreyaciendo al Macizo de Chiapas (Sánchez-Núñez et al., 2015).

Weber et al. (2005), a través de la datación de circones $(\mathrm{U}-\mathrm{Pb})$ contenidos en rocas metamórficas e ígneas del Macizo de Chiapas, determinaron la existencia de un evento tectonotermal con una edad Pérmico-Triásico. Las edades obtenidas en ortogneis fueron $250.9 \pm 2.3 ; 258.4 \pm 1.9 ; 250 \pm$ 16 y $252 \pm 17$ Ma y para circones obtenidos en metasedimentos fue de $241 \pm 14 \mathrm{Ma}$. La variación de edades puede atribuirse a las diferencias en tiempo del evento termal de alto grado, al predominio de muestras de rocas metamórficas sobre las ígneas o quizá a la pérdida de $\mathrm{Pb}$ secundario. Estos resultados coinciden con las edades de 254 $\pm 10 \mathrm{Ma}$ (Rb-Sr) de Damon et al. (1981) y $214 \pm$ 11 a $244 \pm 12$ Ma de Schaaf et al. (2002). De esta forma Weber et al. (2008) concluyen que las rocas metasedimentarias y metaígneas del Complejo Macizo de Chiapas (CMC) de edad Pérmico es el más voluminoso del sureste de México y que constituye el basamento cristalino de la porción sureste del Bloque Maya.

El Macizo de Chiapas está intrusado por dos plutones denominados Tronco de Amatenango de edad Triásico Temprano-Jurásico $(158 \pm 8 \mathrm{Ma})$ y el Tronco de Buenos Aires de edad Jurásico Tardío (154 \pm 12 Ma) (Múgica, 1987) (Figura 2). El primero de estos, está representado por un leucogranito localizado en la porción sur del poblado de Amatenango de la Frontera; un granito porfídico color rosa claro, con fenocristales de feldespatos de 1 a 2 cm de longitud. El segundo plutón está representado por un leucogranito de moscovita de color blanco, que cambia a tonalidades verdes en áreas donde se presenta alteración de las micas por cloritización.

La Formación Todos Santos "Lechos Rojos" de edad Jurásico Tardío-Cretácico Temprano (199 $161 \mathrm{Ma}$ (Godínez, 2009) consta de una serie de conglomerado, arenisca, limolita y lutita que se distribuyen en la porción norte de la cuenca de Motozintla (Moravec, 1983). Los Lechos Rojos descansan en discordancia sobre el Macizo de Chiapas y a su vez, están sobreyacidos por la Formación Sierra Madre del Cretácico (García-Palomo et al., 1987).

Las rocas jóvenes que afloran en la zona de estudio son: lavas andesíticas de edad Plio-Guaternario (Moreno, 1977) localizadas en la porción noroeste 


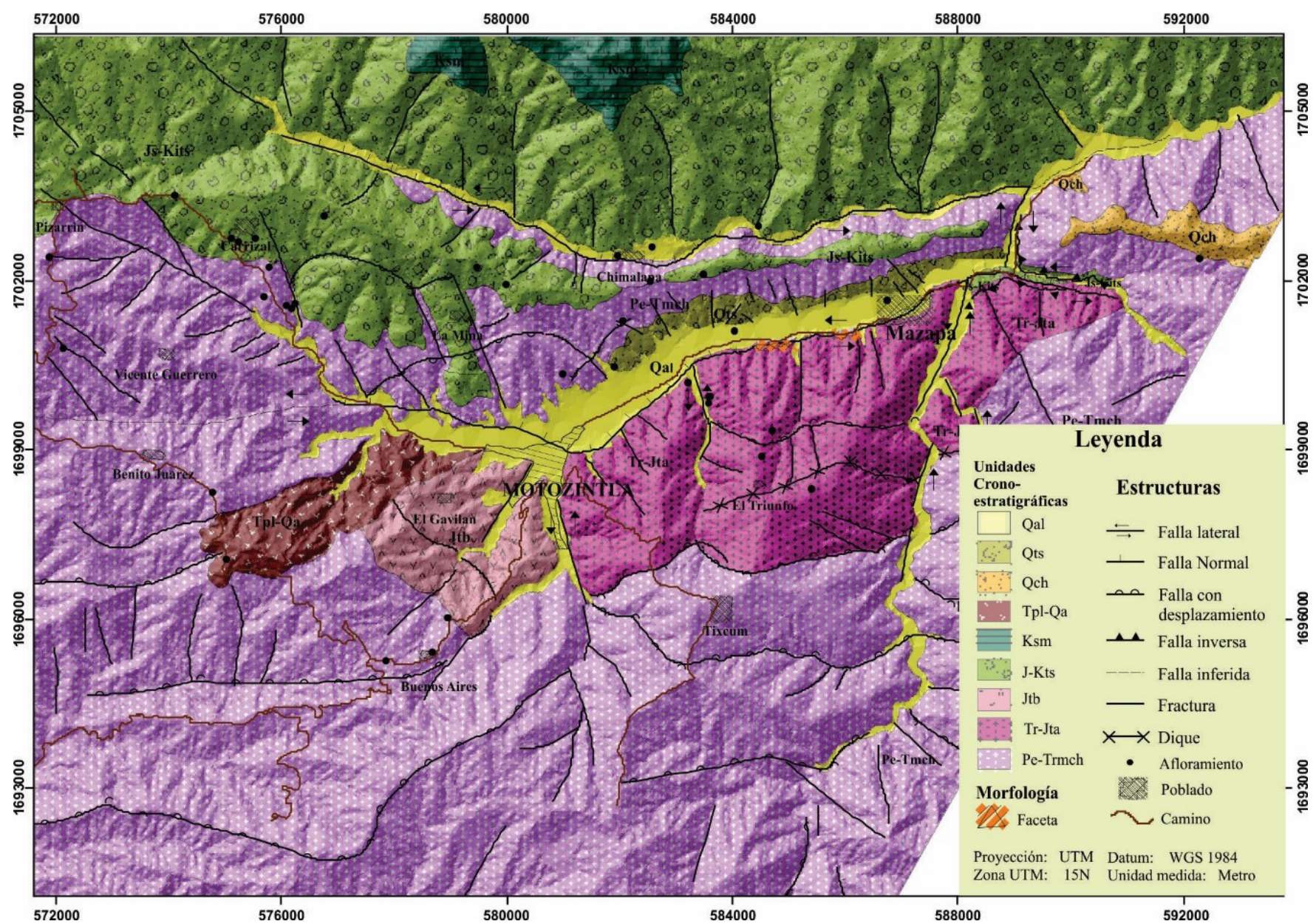

Figura 2 Marco geológico de la región de Motozintla. Las unidades cronoestratigráficas son: Macizo de Chiapas, Pérmico-Triásico (Pe-Trmch); Tronco de Amatenango de la Frontera, Triásico Tardío-Jurásico Temprano (Tr-Jta); Tronco de Buenos Aires, Jurásico Tardío (Jtb); Formación Todos Santos, Jurásico Tardío-Cretácico Temprano (J-Kts); Sierra Madre de Chiapas, Cretácico (Ksm); Lavas Andesíticas, Plioceno-Cuaternario (Tpl-Qa); Flujo Piroclástico Chocoyos, Cuaternario (Qch); Depósito Todos Santos, Cuaternario (Qts); Aluvión, Cuaternario (Qa). Tomada de Sánchez-Núñez et al. (2012).

del poblado de Motozintla; y el flujo piroclástico "Los Chocoyos" con una edad de 84000 años, asociado a la caldera de Atitlán en Guatemala considerada la mayor unidad piroclástica de composición silícea $\left(\mathrm{SiO}_{2}\right.$ : 75 \%) de Centro América y está compuesta por dos miembros, una tefra en la base y un flujo piroclástico en la cima (Drexler et al., 1980; Rabek et al., 1985; Walker, 2006) (Figura 2). Los piroclastos de la caldera se distribuyen en las partes altas de la cuenca, al este de la zona de estudio, sobre el camino que comunica la comunidad de Motozintla de Mendoza, Mazapa de Madero y Amatenango de la Frontera. Con base en inferencias, esta unidad cubrió un área aproxi- mada de $7500 \mathrm{~km}^{2}$, con un volumen aproximado de $200 \mathrm{~km}^{3}$. Finalmente, se presenta un depósito de caída de ceniza, color blanco, originado por una erupción de tipo pliniano del Volcán Santa María en Guatemala en 1902 (Williams y Self, 1983; Walker, 2006).

\section{Metodología}

Para el estudio granulométrico de los abanicos aluviales se identificaron 57 depósitos, asociados a 30 secciones estratigráficas. En los afloramientos estratificados se tomó una muestra por cada capa, 
cuando los estratos eran muy gruesos ( $>1.5 \mathrm{~m})$, el muestreo se efectuó socavando un canal vertical de cima a base del estrato, con la finalidad de obtener una muestra homogénea y representativa. El análisis realizado a las muestras de sedimento fue la distribución granulométrica total (DGT), componentes totales, y la forma de las partículas. Estos se llevaron a cabo en el laboratorio de sedimentología del Instituto de Geofísica de la Universidad Nacional Autónoma de México (UNAM).

Para evaluar la DGT se utilizaron tres técnicas diferentes: (1) para las zonas inaccesibles de los depósitos y/o los sedimentos de grandes dimensiones $(\geq-9 \phi)$, que no pueden ser muestreados por su gran tamaño, se utilizó el procedimiento propuesto por Rosiwal (1898), que consiste en tomar fotografías de la pared del depósito desde una posición conocida. Por medio de un programa de análisis de imágenes, las fotografías se calibran dimensionalmente, procesándolas digitalmente para obtener un mejor detalle y se les sobreponen líneas horizontales paralelas a diferentes alturas. A lo largo de estas líneas se miden sus intersecciones con los clastos y, utilizando el método de Rosiwal, se obtienen las distribuciones granulométricas en los diferentes niveles del depósito. Comparando este método con el de conteo de puntos (análisis modal), que es ampliamente utilizado y ha sido ya comprobado, el resultado revela que el método de Rosiwal es aún más preciso. Las fracciones analizadas incluyen clastos del tamaño de los bloques hasta los guijarros (-4ф a -9ф); (2) para las partículas del tamaño de los gránulos hasta las fracciones de arena $-2 \phi$ a $4 \phi$ se utilizó la técnica del tamizado en seco con los cedazos a intervalos $1 \phi$; (3) para la fracción más fina (de $4 \phi$ a $9 \phi$; limo y arcilla) se utilizó un sistema de escaneo láser de SPECTREX. Para dar confiabilidad a la DGT de cada depósito, se promediaron los tres últimos valores del análisis por tamizado $(-3 \phi ;-4 \phi ;-5 \phi)$ con los tres primeros valores del análisis de la fracción gruesa $(-3 \phi ;-4 \phi$, $-5 \phi)$; la combinación de los tres métodos anteriores fue útil para obtener el porcentaje en peso de cada fracción individual (Kellerhals y Bray, 1971). Los parámetros estadísticos considerados en el análisis granulométrico son los propuestos por Inman (1952) y Folk (1974), ya que estos proporcionan la medida de tendencia central y dispersión de la distribución granulométrica. Para el cálculo de los diferentes parámetros estadísticos se utilizaron los resultados del tamizado, análisis de la fracción gruesa y el escaneo por láser. Los parámetros determinados fueron: Diámetro promedio de los clastos $(\mathrm{Md} \phi)$ (equivalente a la longitud promedio de las intersecciones con la rejilla, medidos con el método óptico), la Clasificación o Sorting $(\sigma \phi)$, Asimetría (S), Curtosis (KG) y la relación Bloque-Matriz, considerando como matriz a todo el material menor a $-6 \phi$ (64 mm).

Con la finalidad de mostrar el comportamiento estadístico de cada fracción de la muestra y apoyados en los criterios de McManus (1988) y Nichols (2009), los resultados se analizaron con diferentes formas gráficas (i.e. histogramas de frecuencia, curvas acumulativas y curvas de frecuencia para cada fracción, Davis, 1970). Con el análisis detallado de las subpoblaciones de partículas, también se lograron inferir las condiciones de evolución y depósito de los materiales.

Para la descripción de las frecuencias de cada depósito se diseñó un formato de ensayo, donde se observó el número de modas en el histograma y se contrastó con el tamaño de la fracción $\phi$. Dependiendo del número de modas se definió si el depósito es unimodal, bimodal o polimodal.

Para generar las curvas de frecuencia acumulativa se utilizó una escala aritmética ordinaria, cuya gráfica tiene forma de "S" en los patrones de distribución normal. Se siguieron los criterios estandarizados para graficar, donde los valores de la fracción gruesa se colocan a la izquierda de la abscisa y la fracción fina a la derecha (McManus, 1988).

\section{Características morfológicas de la cuenca de Motozintla}

La cuenca de Motozintla presenta abanicos aluviales que corresponden a cuerpos formados por 
acumulación de materiales en condiciones subaéreas ocurridos en zonas de ruptura de pendiente, en donde, la parte más baja del sistema fluvial, pertenece a la zona de la planicie aluvial o área de depósito (Nichols, 2009; Bahrami, 2013).

La planicie aluvial de Motozintla forma parte de la cuenca mayor Grijalva-Usumacinta, ubicada en la región hidrológica Frontera Sur y corresponde a la etapa depositacional del río Xelajú Grande (CONAGUA, 2007). Con la interpretación fotogeológica y el procesamiento de imágenes en el Sistema de Información Geográfica (SIG) se definió una red fluvial de tipo subparalelo, donde las corrientes secundarias son paralelas y estas son oblicuas a la corriente principal. Lo anterior indica que el drenaje de la cuenca presenta un marcado control estructural, con fallas y fracturas provocadas por la dinámica del sistema Polochic como se describió anteriormente. La red hidrográfica está desarrollada principalmente en rocas graníticas del macizo de Chiapas y rocas sedimentarias de la Formación Todos Santos.

Las corrientes fluviales más importantes de la cuenca son: el río Xelajú Grande con 21 km de longitud desde su nacimiento a $2500 \mathrm{msnm}$ hasta el poblado de Mazapa de Madero situado a 1060 msnm; y el río Allende y el río Mina con 7.5 y 4.7 $\mathrm{km}$ de longitud, respectivamente. Ambos medidos desde su nacimiento hasta su unión con el río Xelajú Grande (Figura 3). Los ríos Mina y Allende constituyen los dos afluentes principales del río Xelajú Grande y sus aportes de sedimentos incrementan significativamente el volumen de los depósitos en la planicie aluvial (Figura 3).

La cuenca está dominada por las corrientes fluviales que llegan paralelas a las corrientes principales. De acuerdo con De Pedraza (1996), se pueden clasificar como de canales rectilíneos que poseen un índice de sinuosidad bajo (menor a 1.5). Estas corrientes poseen gran capacidad de arranque y

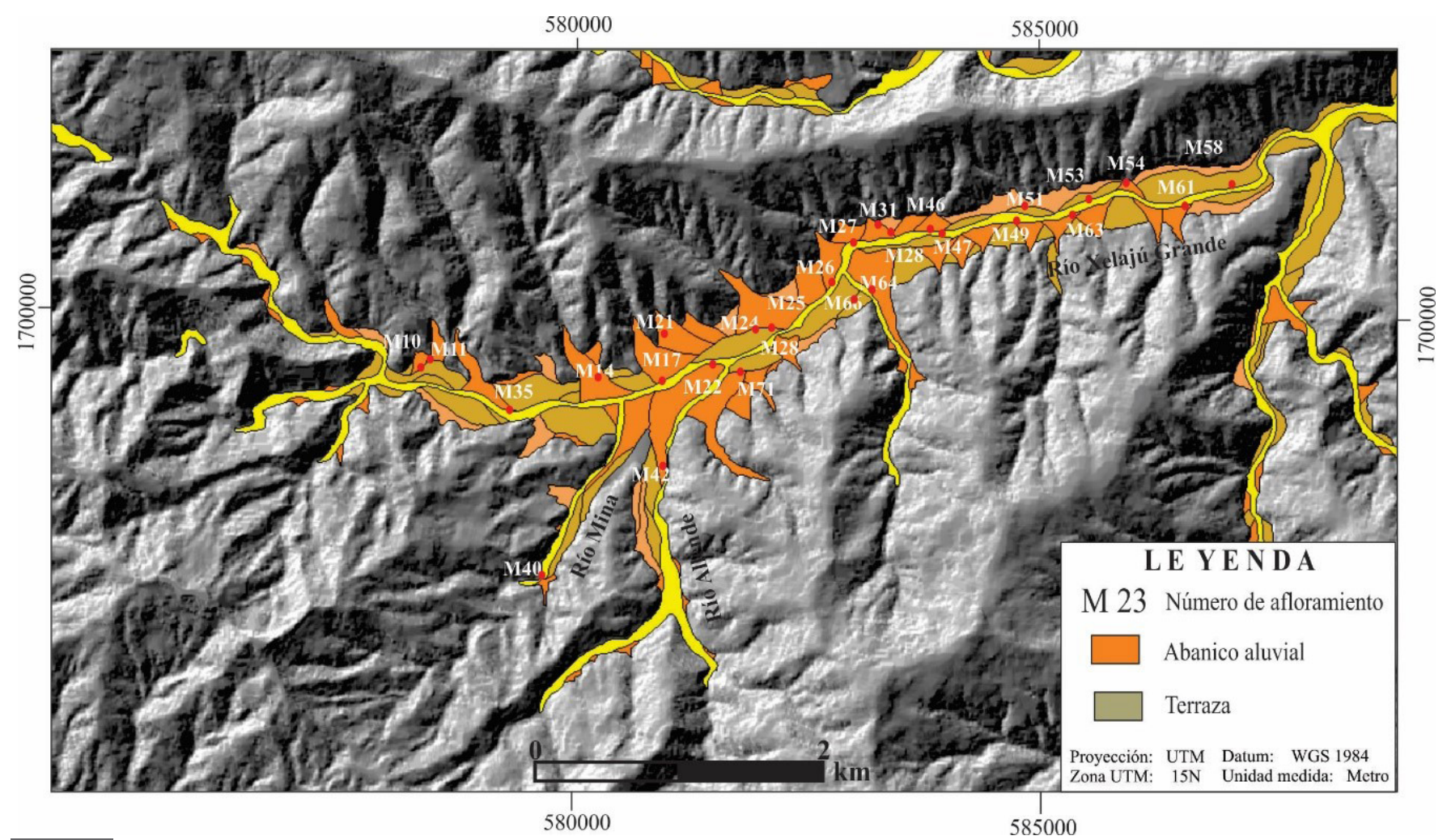

Figura 3 Modelo digital de elevación de la cuenca de Motozintla, mostrando la cartografía de los abanicos aluviales y terrazas. Los números muestran la localización de las muestras recuperadas sobre la planicie aluvial. 
arrastre de materiales por haberse desarrollado en zonas de pendiente elevada $\left(>30^{\circ}\right)$. Por esta razón, se consideran corrientes con gran competencia, ya que pueden transportar carga de fondo y suspensión, llegando a movilizar grandes bloques.

La condición de estabilidad geomórfica de la cuenca ha sido afectada por perturbaciones tectónicas consideradas como procesos de largo plazo. También se presentan afectaciones de corto plazo, representadas por acciones antrópicas y por procesos climáticos de origen hidrometeorológico que provocan lluvias extraordinarias e inundaciones como lo ha descrito Harvey (2007) para otras regiones del mundo. En la cuenca de Motozintla se pone de manifiesto que el sistema fluvial es sensible a los cambios tectónicos, climáticos y litológicos; los que inducen cambios en la topografía debido a las diferencias en la tasa de incisión de los ríos, así como en las laderas del sistema montañoso (Castillo et al., 2015).

En los afloramientos de la planicie aluvial del río Xelajú Grande se observan depósitos con dos tipos de acreción: una vertical, desarrollada por la sedimentación y crecimiento por sobreposición de sedimentos (Gutiérrez-Elorza, 2008; Allen, 1970); y una acreción lateral, resultado de la migración de los canales a través de la planicie y por el depósito de sedimentos en eventos extraordinarios de inundación como los ocurridos en 1998 y 2005. Los depósitos sobre la planicie aluvial del río Xelajú Grande formaron abanicos aluviales y terrazas de grandes dimensiones que se extienden por más de 13 km (Caballero et al., 2006; Sánchez-Núñez et al., 2015). Hoy en día estos depósitos forman los cimientos de la cabecera municipal de Motozintla, donde habitan más de 23755 personas (SEDESOL, 2017). Este escenario pone en evidencia el peligro al que se expone la población ante el depósito de detritos sobre la planicie (Figura 4a).

Debido a la actividad tectónica de la falla Polochic los depósitos de la planicie aluvial se encuentran seccionados, presentando cortes verticales de hasta $25 \mathrm{~m}$. Este seccionamiento pudo haber sido producido por actividad sísmica a lo largo de la falla Polochic (Sánchez-Núñez et al., 2015). Estos cortes permitieron observar y muestrear los diferentes eventos de depósito que se presentan en este trabajo (Figura 4b). El último gran sismo registrado a lo largo de la falla Chixoy-Polochic ocurrió al este de Motozintla en territorio Guatemalteco
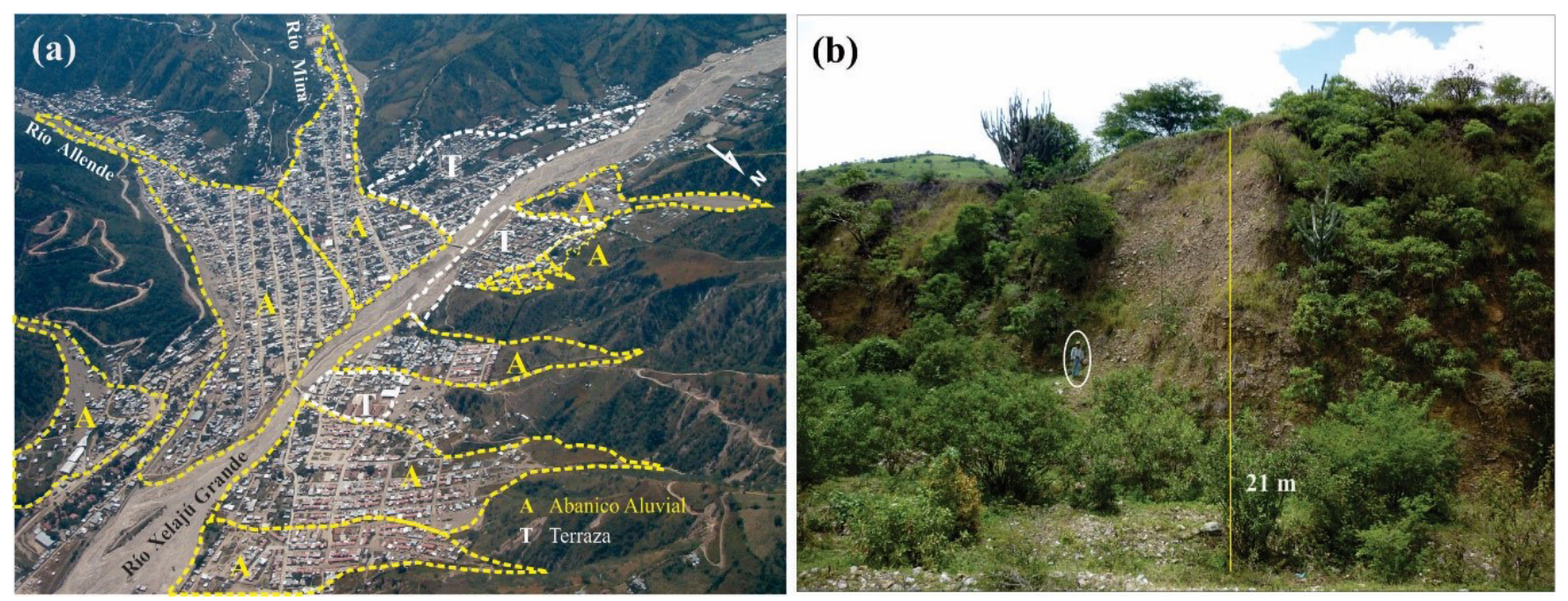

Figura 4 (a) Vista aérea de la ciudad de Motozintla. Se muestra la morfología de los depósitos de abanicos aluviales, emplazados en la planicie del río Xelajú Grande. Nótese el enorme flujo de escombros generado por las precipitaciones pluviales extremas causadas por el huracán "Stan" en 2005. Fotografía cortesía de V.H. Robledo. (b) corte vertical del abanico aluvial (M26), sobre la traza de la falla Polochic. Se muestra un depósito masivo de flujos de escombros con textura soportada por matriz y esporádicos lentes de arena gruesa, la persona mide $1.68 \mathrm{~m}$ de altura. 
el 22 de julio de 1816 (Mw of 7.5 - 7.75), el cual tuvo una profundidad de ruptura de $10 \mathrm{~km}$ y un área afectada de $13000 \mathrm{~km}^{2}$ (White, 1985; Peraldo y Montero, 1999).

\section{Resultados}

\subsection{GARAGTERÍSTICAS GRANULOMÉTRICAS Y SEDIMENTOLÓGIGAS DE LOS ABANICOS ALUVIALES}

Los resultados de los 57 análisis granulométricos se presentan en las figuras 5 y 6 , en la Tabla 1 y en el

\section{M53}

M35

용-

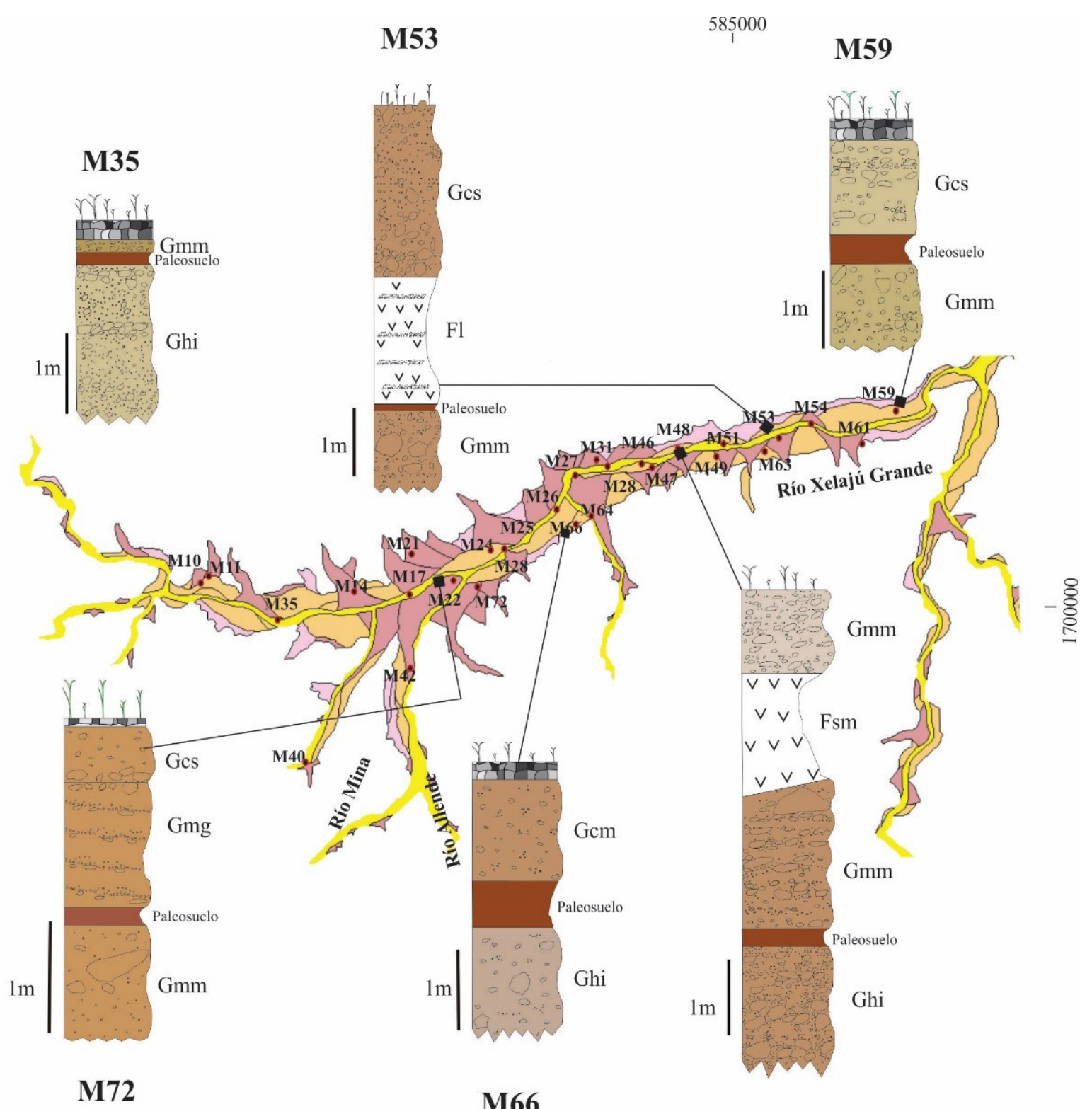

$\stackrel{1}{580000}$

M48

Figura 5 Se muestran ejemplos de seis secciones estratigráficas sobre la planicie aluvial de la cuenca de Motozintla, señalando las facies principales que se identificaron en cada una de estas. 


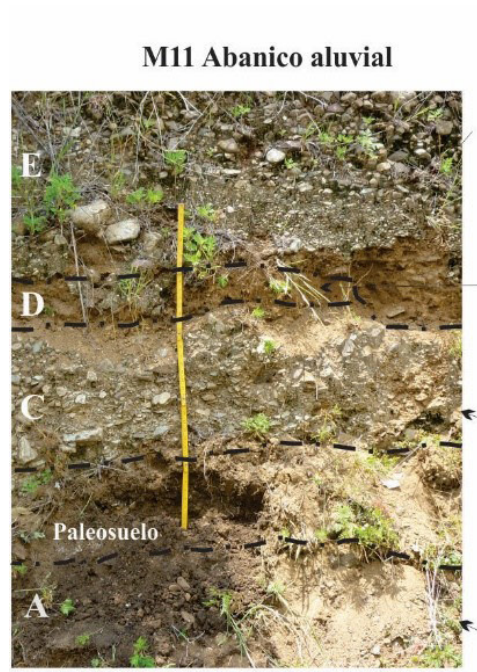

(a)
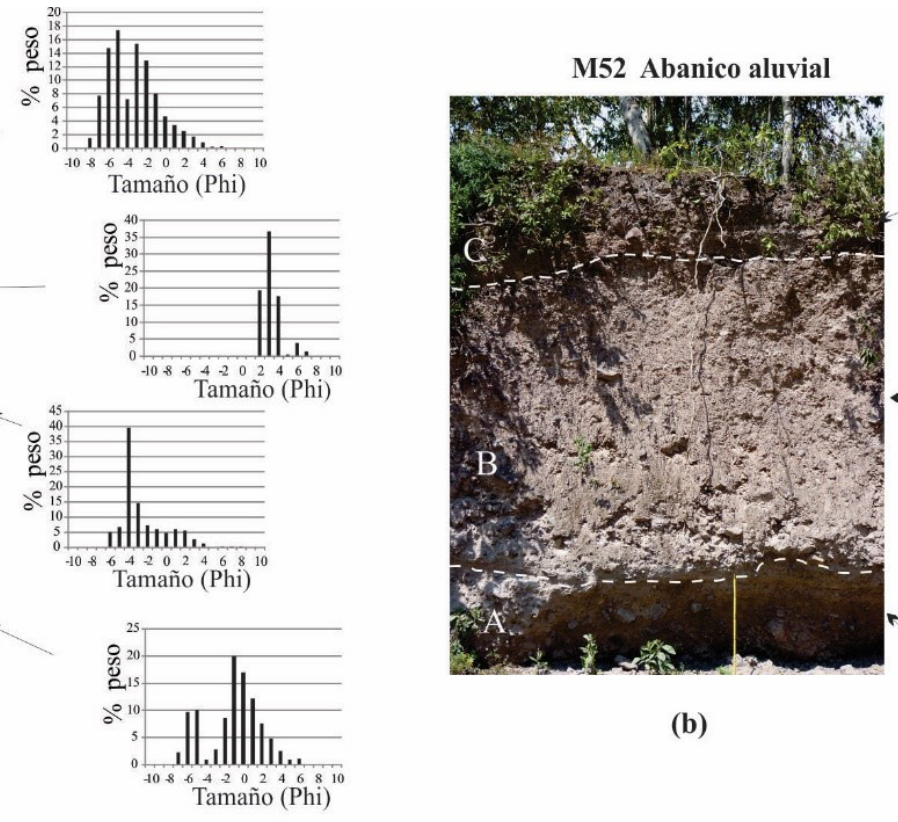

(b)
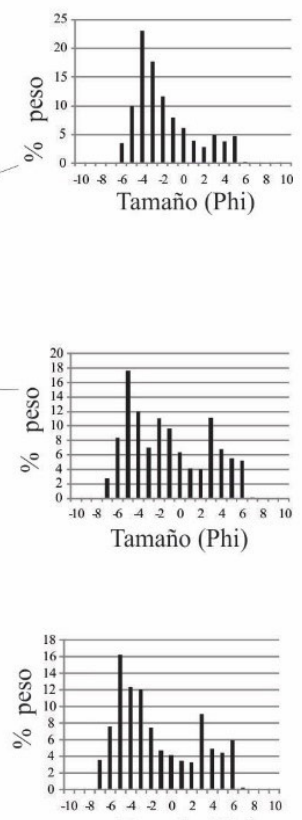

Tamaño (Phi)

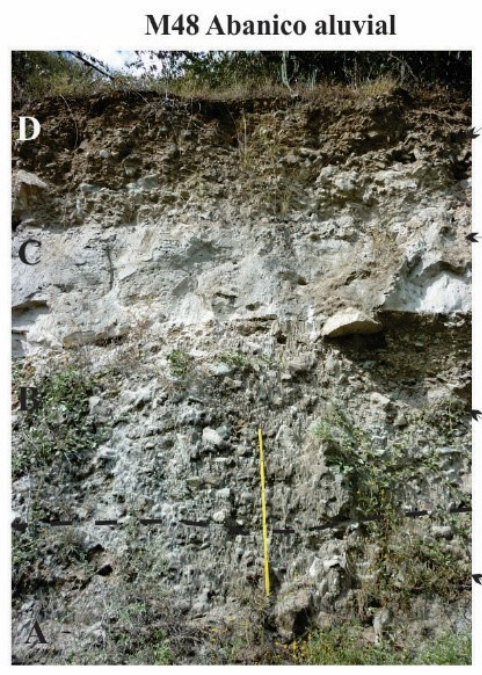

(c)
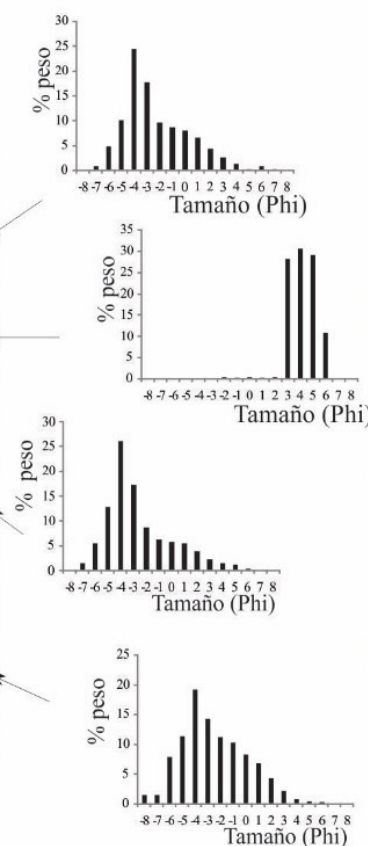

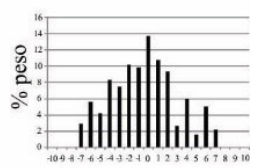

M68 Abanico aluvial Tamaño (Phi)

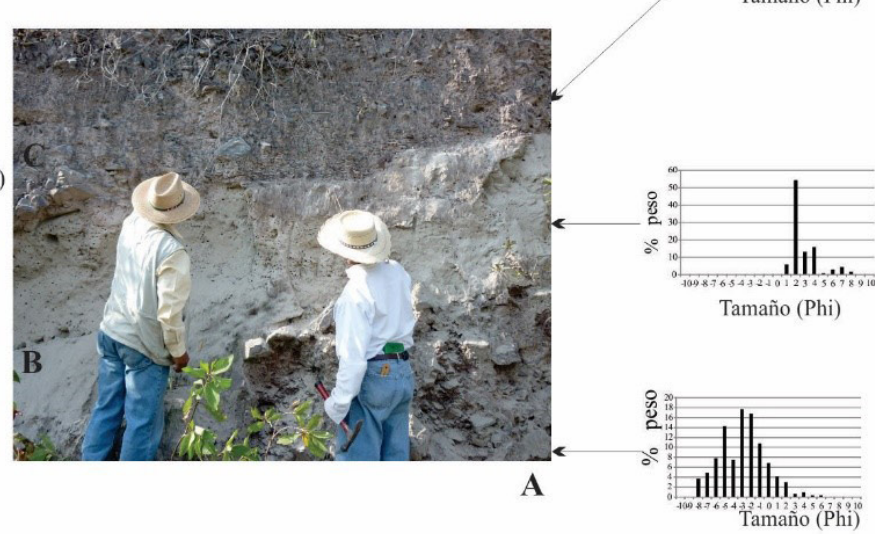

(d)

Figura 6 Histogramas de frecuencia de tamaños de partícula de depósitos de flujos de escombros en abanicos aluviales de la cuenca de Motozintla. (a) sección M11, el afloramiento muestra depósitos de flujos de escombros separados por paleosuelos. (b) sección M52, se observa un depósito masivo con clastos grandes (Moda en - $5 \varphi$ ), soportados por una matriz compacta y endurecida. (c) sección M48 y (d) sección M68, muestran depósitos estratificados con variación textural por tratarse de flujos piroclásticos retrabajados. 
Tabla 1. Facies sedimentarias principales identificadas en los depósitos de abanicos aluviales en la cuenca de Motozintla, Chiapas. Elaborada con criterios de Miall $(2000,2006)$ y Neves et al. (2005).

\begin{tabular}{|c|c|c|c|c|c|}
\hline Código & & Facies & $\begin{array}{l}\text { Estructuras } \\
\text { sedimentarias }\end{array}$ & $\begin{array}{l}\text { Textura y } \\
\text { fábrica }\end{array}$ & Interpretación \\
\hline $\mathrm{Gmm}$ & \multirow{4}{*}{ 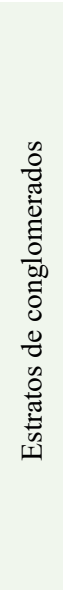 } & $\begin{array}{l}\text { Conglomerado/soportados } \\
\text { por matriz, ocasionales } \\
\text { lentes de arena }\end{array}$ & Masivo & \multirow{4}{*}{ 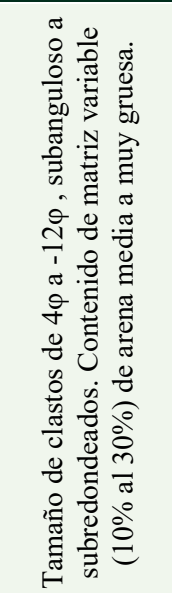 } & $\begin{array}{c}\text { Flujos de escombros } \\
\text { Flujos de gravedad, mal clasificado, } \\
\text { compactos, asociados con abanicos } \\
\text { aluviales. }\end{array}$ \\
\hline Gcs & & $\begin{array}{l}\text { Conglomerado soportado por } \\
\text { matriz }\end{array}$ & $\begin{array}{l}\text { Estratificado con } \\
\text { gradación normal }\end{array}$ & & $\begin{array}{l}\text { Flujos de escombros, mal clasificado. } \\
\text { Asociados con abanicos aluviales. }\end{array}$ \\
\hline Ghi & & $\begin{array}{l}\text { Conglomerado masivo, } \\
\text { soportado por matriz }\end{array}$ & $\begin{array}{l}\text { Masivo con } \\
\text { imbricación }\end{array}$ & & $\begin{array}{c}\text { Flujos de escombros, mal clasificado, } \\
\text { compactación media-alta } \\
\text { Asociados con abanicos aluviales. }\end{array}$ \\
\hline Gh & & $\begin{array}{c}\text { Conglomerado soportado por } \\
\text { granos }\end{array}$ & Masivo & & $\begin{array}{c}\text { Flujos de gravedad, mal clasificado, poco } \\
\text { compactos, asociados con abanicos } \\
\text { aluviales. }\end{array}$ \\
\hline $\mathrm{Bmm}$ & \multirow{4}{*}{ 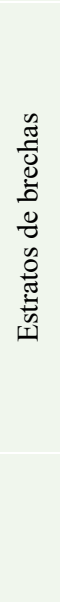 } & $\begin{array}{l}\text { Brecha soportada por matriz, } \\
\text { ocasionales lentes de arena }\end{array}$ & Masiva & \multirow{4}{*}{ 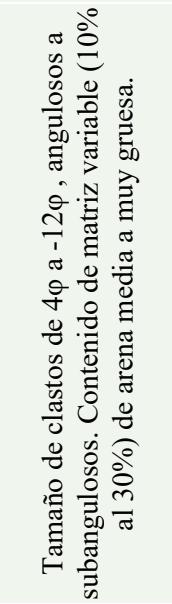 } & $\begin{array}{l}\text { Flujos de escombros, flujos de gravedad, } \\
\text { mal clasificada, compactas, asociadas con } \\
\text { abanicos aluviales. }\end{array}$ \\
\hline Bes & & Brecha soportada por matriz & $\begin{array}{l}\text { Estratificada con } \\
\text { gradación normal } \\
\text { incipiente }\end{array}$ & & $\begin{array}{l}\text { Flujos de escombros, mal clasificada. } \\
\text { Asociadas con abanicos aluviales. }\end{array}$ \\
\hline Bhi & & $\begin{array}{l}\text { Brecha masiva, soportada } \\
\text { por matriz }\end{array}$ & $\begin{array}{l}\text { Masiva con } \\
\text { imbricación } \\
\text { incipiente }\end{array}$ & & $\begin{array}{c}\text { Flujos de escombros, mal clasificada, } \\
\text { compactación media-alta } \\
\text { Asociadas con abanicos aluviales. }\end{array}$ \\
\hline $\mathrm{Bh}$ & & Brecha soportada por granos & Masiva & & $\begin{array}{c}\text { Flujos de gravedad, mal clasificada, poco } \\
\text { compactas, asociadas con abanicos } \\
\text { aluviales. }\end{array}$ \\
\hline $\mathrm{Gcm}$ & \multirow{2}{*}{ 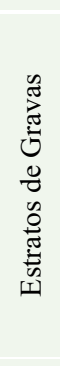 } & Gravas & Masivo & \multirow{2}{*}{ 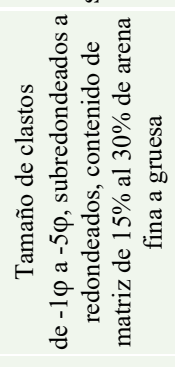 } & $\begin{array}{l}\text { Flujos de escombros, } \\
\text { mal clasificados, poco compactos, } \\
\text { asociados con abanicos aluviales. }\end{array}$ \\
\hline Gmg & & $\begin{array}{c}\text { Gravas soportadas por } \\
\text { matriz }\end{array}$ & $\begin{array}{c}\text { Estratificación } \\
\text { horizontal con } \\
\text { imbricación. Y } \\
\text { ocasional } \\
\text { estratificación } \\
\text { cruzada. }\end{array}$ & & $\begin{array}{l}\text { Flujos de gravedad, mal clasificado, } \\
\text { compactación media-alta, asociados con } \\
\text { abanicos aluviales. }\end{array}$ \\
\hline Fal & \multirow{3}{*}{ 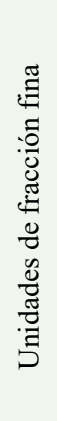 } & $\begin{array}{l}\text { Láminas horizontales de } \\
\text { arena fina, limo y arcilla }\end{array}$ & $\begin{array}{l}\text { Laminación } \\
\text { horizontal. }\end{array}$ & \multirow{3}{*}{ 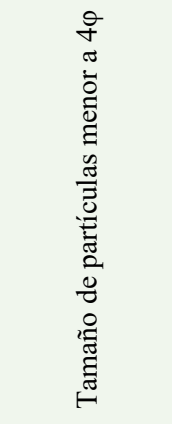 } & $\begin{array}{c}\text { Flujos hiperconcentrados, retrabajo de } \\
\text { flujos piroclásticos (mal clasificados), } \\
\text { muy poco compactos }\end{array}$ \\
\hline Fmp & & Limo, arcilla, piroclástico & $\begin{array}{l}\text { Laminación } \\
\text { horizontal fina, } \\
\text { gradación normal, } \\
\text { inversa o ambas. }\end{array}$ & & $\begin{array}{l}\text { Flujos hiperconcentrados, retrabajo de } \\
\text { flujos piroclásticos, bien clasificados, } \\
\text { muy poco compactos }\end{array}$ \\
\hline $\mathrm{Fc}$ & & Ceniza & $\begin{array}{l}\text { Estratificación } \\
\text { horizontal, } \\
\text { gradación normal. }\end{array}$ & & Depósito de caída piroclástica. \\
\hline
\end{tabular}


Anexo 1. La distribución granulométrica de estos depósitos indica que se trata de 23 depósitos con curvas unimodales, 20 bimodales y 14 polimodales. En la Tabla 1 se describen las características de las facies sedimentarias principales de cada depósito. Se seleccionaron las secciones estratigráficas más completas y representativas de los depósitos en los abanicos aluviales que fueron M11, M52, M48 y M68 (Figura 6).

Para la descripción de los depósitos de abanico aluvial se seleccionó la sección M11. Esta sección se localiza en el extremo oeste de la planicie aluvial, sobre el cauce del río Xelajú Grande (Figura 3). Consta de cuatro depósitos de flujos de escombros (FE) y un paleosuelo intercalado (Figura 6a). Los histogramas de frecuencias indican que la capa (A) es bimodal con modas en $-5 \phi$ y $-1 \phi$; la capa (B) es un paleosuelo fechado en $1250 \pm 60$ años AP (Sánchez-Núñez et al., 2015); la capa (C) es unimodal con moda en -4申; la capa (D) es bimodal, con modas en $3 \phi$ y $6 \phi$; y la capa $(\mathrm{E})$ tiene una distribución bimodal, con modas en $-5 \phi$ y $-3 \phi$. En general, se observa que se tienen dos poblaciones significativas.

Todos los depósitos (A, C, D, E) presentan una mala selección, con un promedio de Diámetro Medio de 2.2 y desviación estándar $(\sigma \phi)$ de: $\mathrm{A}=$ $2.95, \mathrm{C}=2.53, \mathrm{D}=3.04$ y $\mathrm{E}=2.74$. Estos valores varían de acuerdo con el depósito, pero se mantienen en un rango granulométrico similar a los diferentes depósitos que conforman los abanicos aluviales. Estos depósitos presentan una asimetría positiva leptocúrtica: $(\mathrm{A})(\mathrm{KG}=1.12) ;(\mathrm{C})(\mathrm{KG}=$ 1.12); (D) $(\mathrm{KG}=1.11)$ y $(\mathrm{E})(\mathrm{KG}=2.3)($ Figura 6). A partir del análisis de las características texturales y granulométricas de los depósitos resumidos en la Tabla 1 y empelando los criterios de Luzón (1999) podemos definir que se trata de conglomerado y brecha sostenidos por matriz arenosa, heterolitológicos y heterométricos, con variación milimétrica a decimétrica. Los depósitos presentes en la planicie de Motozintla se pueden clasificar como detritos emplazados en abanicos aluviales de alta eficacia de transporte a partir de flujos acuosos no confinados de alta energía. Estas características sugieren que el transporte de sedimentos se llevó a cabo a través de corrientes hídricas ocasionadas por precipitaciones pluviales extraordinarias (anómalas), asociadas a huracanes o tormentas tropicales. Su canal principal está encajado y confinado hasta llegar a la zona de ruptura de pendiente (ápice), donde la carga de sedimentos (con predominio de la fracción gruesa) se libera con una traza de dispersión radial. Cuando estos depósitos presentan horizontes arenosos, frecuentemente lenticulares, se asocian a episodios de depósito con baja tasa de descarga.

\subsection{GURVAS DE FREGUENGIA AGUMULATIVA Y EN ESGALA PROBABILÍSTIGA}

El uso de curvas acumulativas permitió la comparación directa entre varias muestras y brindó la oportunidad de comparar las fracciones granulométricas de los depósitos típicos de abanico aluvial (Figura 7).

En la Figura 7a se observa un comportamiento granulométríco homogéneo de la fracción media y una acumulación importante de la fracción gruesa a partir de $-4 \phi$ a $-9 \phi$. Los depósitos forman curvas con pendiente que varía entre $35^{\circ}$ y $60^{\circ}$, pero con un cambio gradual entre las diferentes subpoblaciones, mostrando que la mayoría de las partículas se encuentran en el intervalo de $-5 \phi$ a $2 \phi$, mientras que la cola de las partículas finas inicia en la fracción $2 \phi$. Esta cola se extiende hasta $8 \phi$, debido a la presencia de cenizas volcánicas retrabajadas, correspondientes al flujo piroclástico Chocoyos, los que forman depósitos de flujos hiperconcentrados. De la misma forma se muestra el gráfico en escala probabilística (Figura 7b) que nos permite entender con mayor claridad el significado de los diferentes tamaños de fracciones y su relación con el proceso depositacional (Visher, 1969). Lo más relevante de este gráfico es que nos permite identificar y separar los distintos puntos de inflexión de la curva y los cambios de pendiente, permitiendo el reconocimiento de cada segmento de curva. El gráfico presenta el comportamiento de dos flujos de escombros (M11c y M48a), facies Gmm y Ghi, donde se aprecia que la fracción gruesa $(1 \phi$ a $-5 \phi)$ 


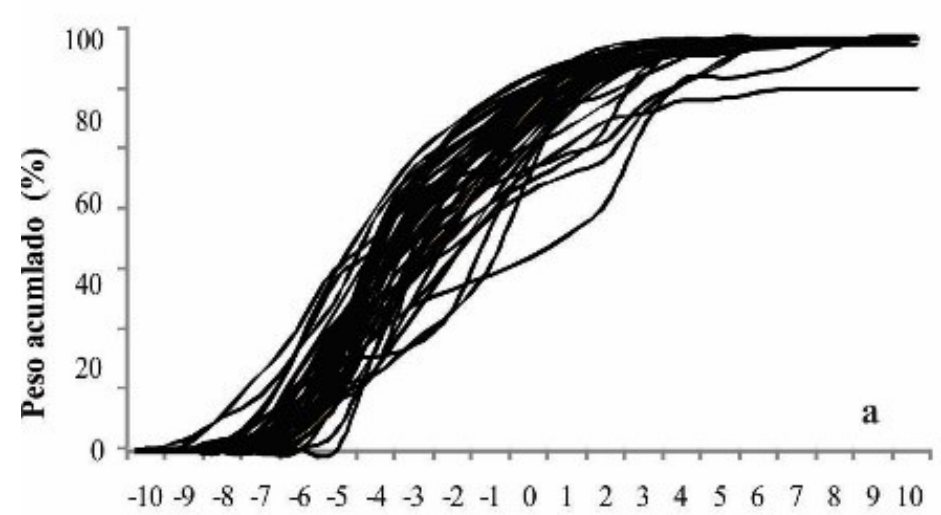

Diámetro Medio (MdF)

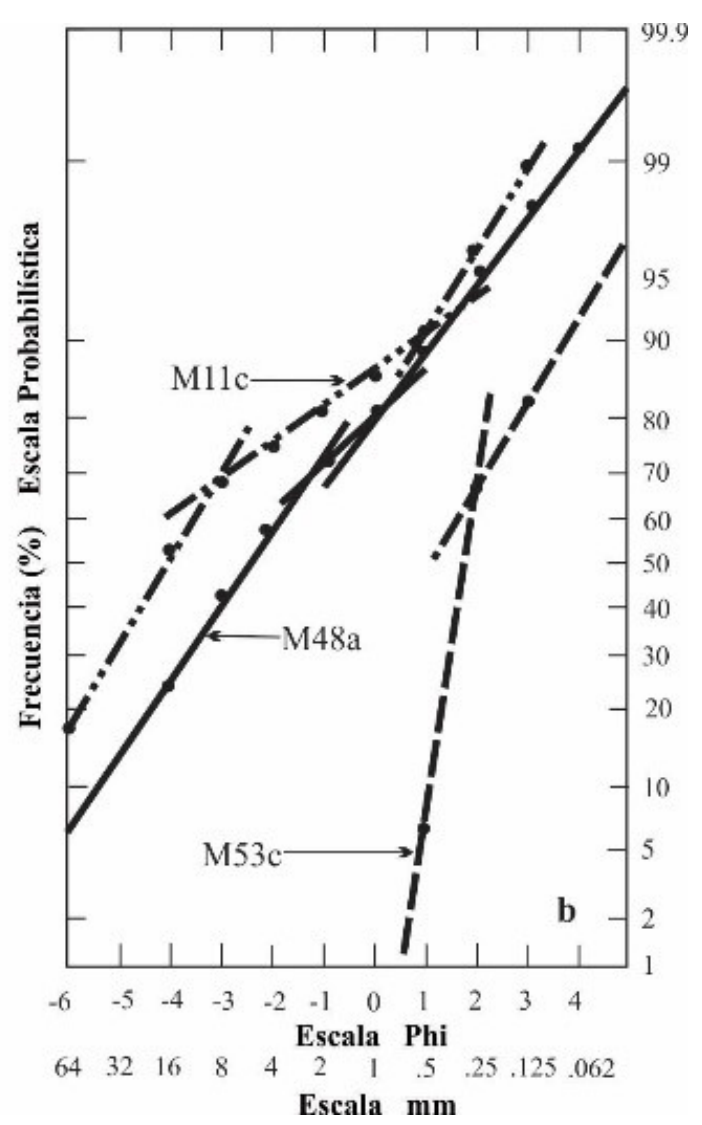

Escala mm

Figura 7 a) Curvas acumulativas de los depósitos en abanicos aluviales, b) curvas en escala probabilística de flujos de escombros (M1 1 c y M48a) y flujo hiperconcentrado (M53c) en la planicie aluvial de la cuenca de Motozintla, Chiapas.

se desplazó por un proceso de tracción y en menor proporción, parte de fracción gruesa y la fracción $1 \phi$ a $3 \phi$ se movilizó por un proceso de saltación. Finalmente, una pequeña porción de la fracción fina fue transportada en suspensión. Contrastando con los gráficos anteriores, se observa la curva M53c, facies Fl, cuyo transporte se desarrolló casi en su totalidad por saltación y suspensión, debido al tamaño de las partículas que forman el depósito $(1 \phi$ a $4 \phi)$.

Los rasgos específicos del sistema aluvial en la cuenca de Motozintla están definidos principalmente por la morfología de la cuenca, el volumen de agua disponible, la cantidad y tipo de sedimentos, naturaleza de las áreas fuente y el grado de pendiente de la superficie de depósito (Nichols, 2009). Otros factores que intervienen en este sistema son el fuerte control estructural (frente de montaña), el clima y las características del patrón de drenaje (Figura 8).

Retomando los criterios de Basu y Sarkar (1990), sobre la naturaleza de los sedimentos presentes en abanicos aluviales, se puede mencionar que los depósitos predominantes en la zona de estudio por lo general son de grano grueso, mal clasificados e inmaduros, constituidos por gravas, guijarros, cantos y bloques con cantidades subordinadas de arena, limo y arcilla.

En cuanto a los procesos de depósito, se identificaron diferentes estructuras con características sedimentológicas específicas: depósitos de flujos de escombros subaéreos, representados por conglomerados soportados por una matriz fina, aflorando en forma de estratos con muy mala clasificación, alineándose en forma paralela al flujo basal, o bien los clastos presentan orientación al azar (Boggs, 
2006). Sin embargo, los clastos pueden presentar imbricación, pero generalmente no desarrollan estructuras sedimentarias. Pueden existir clastos de dimensiones métricas al interior del flujo y poseer espesores de estrato de unos cuantos centímetros a varios metros (Figura 5).

Otro tipo de depósito identificado en los abanicos, son estratos de gravas gruesas a finas con arenas soportados por una matriz de limos o arcillas bien estratificadas de espesor variable. Es común la imbricación de clastos, llegando a presentar estratificación cruzada de bajo ángulo y también pueden formar antidunas (estructura muy escasa). Los de- pósitos están pobremente clasificados; los estratos suelen presentar gradación normal debido a la disminución de energía de la corriente.

Finalmente, se identificaron depósitos con desarrollo de laminaciones paralelas, llegando a formar estratos que se acuñan, presentando esporádicamente estratificación cruzada y clastos imbricados. Tomando en consideración las características del sistema fluvial en la cuenca de Motozintla y los criterios propuestos por Miall (2000, 2006), se definieron los tipos de facies sedimentarias principales (Tabla 1), para ello se tomaron en cuenta los atributos de los depósitos en términos de los procesos,

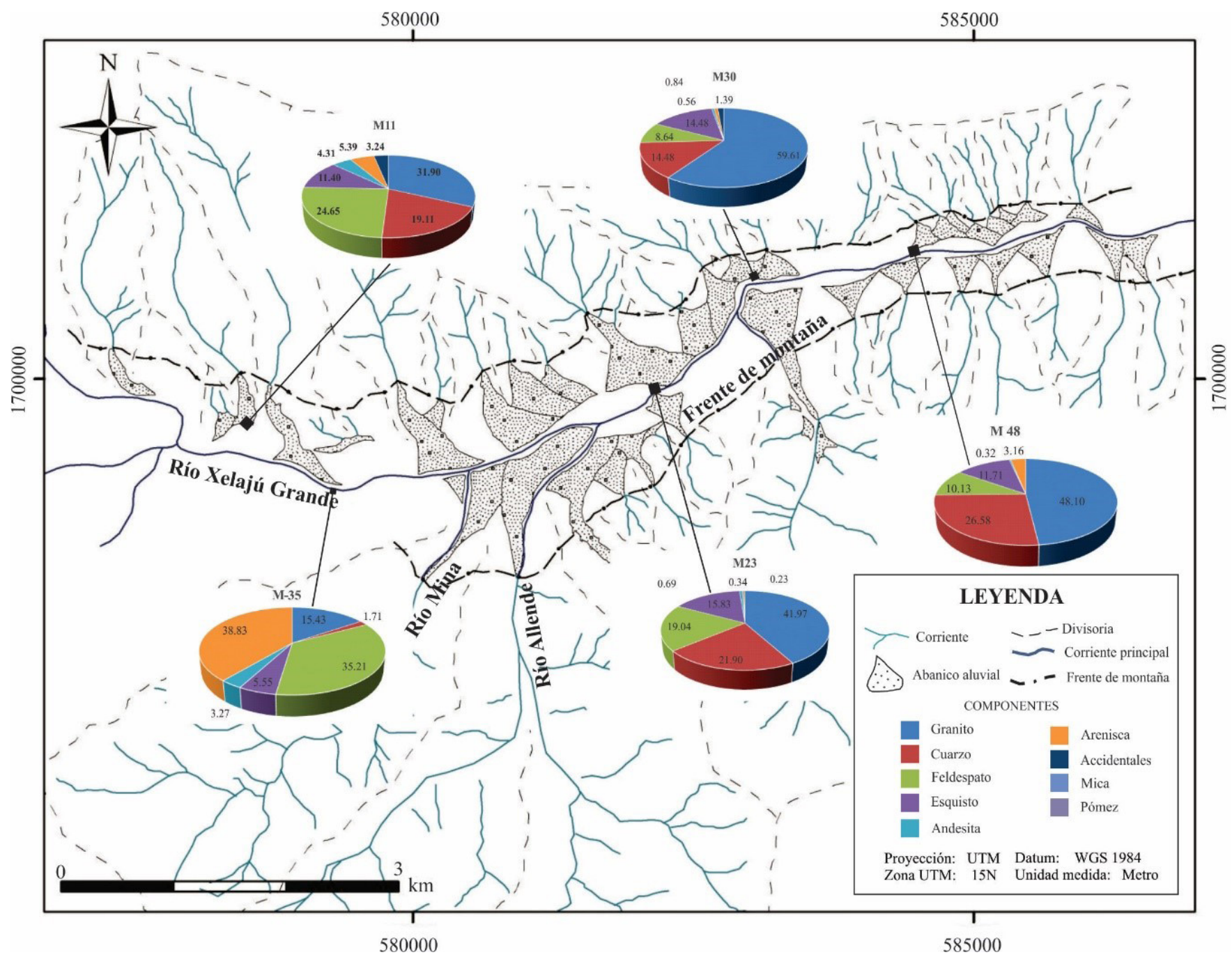

Figura 8 Se muestra la configuración de la red fluvial, las divisorias que enmarcan las subcuencas y los abanicos aluviales como respuesta al transporte de los diferentes tipos de sedimentos (componentes) que provienen de las fuentes de aporte. Modificada de Sanchez-Núñez et al. (2015). 
es decir, tomando en consideración rasgos como el tamaño de las partículas, la composición (Figura 8), características de estratificación y las estructuras sedimentarias.

Con base en los recorridos de campo, los resultados del análisis granulométrico y la definición de las facies mayores, se observa que la mayoría de los depósitos que conforman los abanicos aluviales fueron desarrollados por flujos de escombros.

\section{Discusión}

Para un mejor entendimiento de la génesis de los depósitos es necesario conocer su historia, partiendo de su origen, transporte y su sedimentación (Carrera y Capra, 2008; Capra et al., 2006). Para ello se analizaron las diferencias y similitudes entre los estratos de las secciones estratigráficas, se identificaron las poblaciones más importantes y las muestras con patrones polimodales se dividieron en subpoblaciones (Ashley, 1978). Con los criterios anteriores y los resultados obtenidos del programa SFT (Wohletz et al., 1989) se elaboró la gráfica de la Figura 9, donde se compara el par estadístico del Diámetro Medio $(\mathbf{M d} \phi)$ y la Desviación Estándar $(\sigma \phi)$.

Se identificaron tres grupos de muestras con rangos granulométricos contrastantes. La subpoblación "A", representa el grupo más dominante, estos depósitos presentan en general una selección

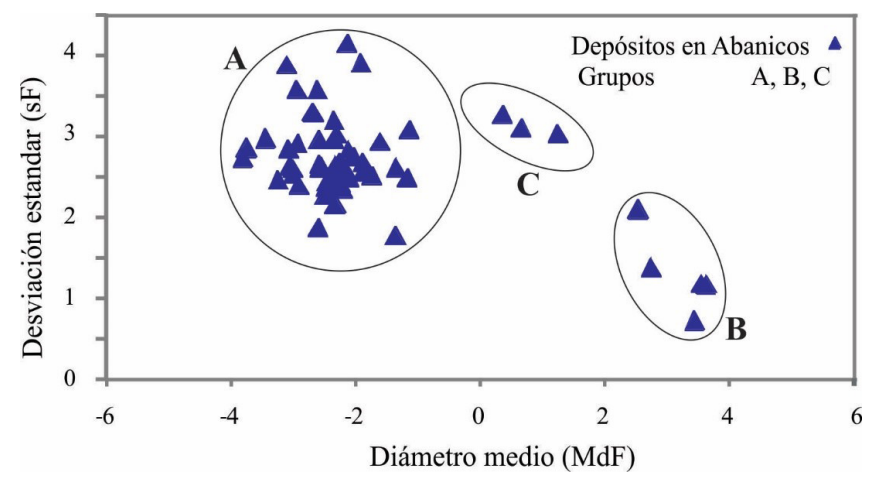

Figura 9 Representación binaria del Diámetro Medio (Md $\varphi)$ vs. Desviación Estándar $(\sigma \varphi)$ con valores estadísticos según Inman (1952) y Folk (1974) para el rango total de partículas en depósitos de flujos de escombros e hiperconcentrados. granulométrica que va de mala a muy mala, con un valor mínimo de $(\sigma \phi)=1.87$ y un valor máximo de $(\sigma \phi)=4.16$; presenta un diámetro medio mínimo de $(\mathrm{Md} \phi)=1.26$ y un valor máximo de $(\operatorname{Md} \phi)=-3.81$. Los datos anteriores corroboran que estos depósitos son flujos de escombros y son muy similares a los resultados obtenidos en el volcán Nevado de Toluca, Jocotitlán y colapsos en el volcán Tacaná (Macías et al., 1997, 2004, 2010); por ejemplo, el estudio comparativo de depósitos epiclásticos en diferentes ambientes volcánicos reportados en el volcán Iztaccíhuatl, Popocatépetl, volcán Santa Elena, entre otros realizados por Carrera y Capra (2008).

La Figura 9, también pone en evidencia una secuencia de muestras que contiene subpoblaciones con granulometría notablemente más fina, subpoblación "B", que contrasta con la población "A". Esto se debe a dos causas principales: la primera es que por lo menos en doce de las treinta columnas estratigráficas en abanicos aluviales, se presentan depósitos de flujos piroclásticos retrabajados estratificados, cuya granulometría es del orden de $4 \phi$. Esta característica se observa en las secciones M48 y M68, donde ocurren depósitos que se caracterizan por presentar laminación horizontal fina (que posee entre el $35 \%$ y $60 \%$ en volumen de sedimento), que corresponde a un depósito de flujo hiperconcentrado $(\mathrm{FH})$ pseudo-estratificado, separando depósitos de conglomerado masivo y flujos de escombros (FE); lo anterior se aprecia en la Figura 6 y en las curvas de frecuencia acumulativas de la Figura 7. Este tipo de depósito es comparable con la matriz de los flujos de escombro de tipo cohesivo reportados por Capra y Macías (2002) en la presa el Naranjo del Nevado de Colima y en los depósitos de flujo de escombro de Pilcaya del Volcán Nevado de Toluca (Capra y Macías, 2000). La segunda causa de la presencia de fracciones finas en los depósitos se debe a eventos de precipitación pluvial intensa y la transformación de los flujos de escombros en flujos hiperconcentrados (Mulder y Alexander, 2001). Este fenómeno también es frecuente en ambientes volcánicos, como es el caso de los depósitos de las terrazas lahári- 
cas en la barranca de Huiloac, al NE del volcán Popocatepetl (Franco-Ramos et al., 2017), donde se presentan flujos de escombros y distancia hasta su depósito (Caballero et al., 2006; Saucedo et al., 2004). Estos depósitos se caracterizan por presentar una mejor selección, con valores de Diámetro Medio $(\mathrm{Md} \phi)=3.65$ como máximo y con Desviación Estándar de $(\sigma \phi)=0.72$ como valor mínimo; estos datos indican una selección moderadamente buena para estos depósitos. Es importante resaltar que la mayoría de los depósitos de flujos hiperconcentrados se presentan en terrazas fluviales, que han quedado descubiertos debido a los procesos erosivos en la planicie aluvial de la cuenca y cuyos depósitos más antiguos tienen una edad aproximada de 17 Ka AP (Sánchez-Núñez et al., 2015).

Los factores detonantes que pudieron generar los flujos de escombros en Motozintla, en los años 1998 y 2005, fueron la topografía abrupta con pendientes mayores a $30^{\circ}$, canales fluviales de corte en "V", materiales erosionados disponibles para alimentar un flujo de escombros y una precipitación inusual. Un ejemplo similar es el estudio de Zhou et al. (2015), quienes realizaron un trabajo experimental del megaflujo de escombros ocurrido en la provincia de Wenjia, China. En ambos casos, se registró una fuerte erosión del sustrato en los canales situados a varios kilómetros aguas arriba, un ensanchamiento de los cauces y un gran depósito de sedimentos en la zona baja de la cuenca, dándole características de alto peligro y alto costo para las regiones donde se emplazaron. En la fase experimental realizada por Zhou et al. (2015), se observan horizontes de granulometría fina $(-1 \phi$ a $4 \phi)$ correspondiente a la facies media del depósito que denominaron "surge of debris flow", que se sobreponen a horizontes con partículas más gruesas $(-2 \phi$ a $-6 \phi)$. Estos depósitos son similares a los flujos hiperconcentrados reportados en este estudio (subpoblación B de este trabajo), que igualmente se sobreponen a horizontes con granulometría más gruesa.

Otro aspecto importante que se aborda en este estudio es la transformación de los flujos de escombros a flujos hiperconcentrados, este proceso de transformación se presenta en Motozintla cuando se unen los sedimentos de los ríos Mina y Allende con los sedimentos del río Xelajú Grande, después de haber recorrido $\sim 7 \mathrm{~km}$ desde la fuente de aporte de sedimentos. La evidencia de este proceso es la presencia de laminación y escasa gradación normal en los sedimentos más finos (Figura 6d). Un ejemplo de este proceso de transformación es el presentado por Saucedo et al. (2008) en la barranca de Atenquique, en las inmediaciones del Volcán Nevado de Colima, México. Finalmente, se tiene la presencia de la subpoblación "C", que presenta características intermedias entre las poblaciones "A" y "B", pudiendo clasificarse como depósitos de transición que ocurre en el proceso de transformación de flujo de escombros a flujo hiperconcentrado (Mulder y Alexander, 2001).

\subsection{GONSIDERAGIONES SOBRE EL PELIGRO GEOLÓGICO EN MOTOZINTLA}

La ubicación geográfica y condiciones geológicas de la cuenca de Motozintla la sitúan como una región de alta vulnerabilidad y expuesta a peligros de diferente naturaleza. Esta condición ha llamado la atención de muchos investigadores que han realizado estudios de corte geológico y social como los realizados por Caballero et al. (2006), Carballido (2008), Briones (2010), Novelo et al. (2013), entre los principales. Este trabajo está enfocado al estudio de los abanicos aluviales y las características granulométricas y sedimentológicas de los flujos de escombro que los conforman. Esto resulta muy importante debido a que el registro geológico indica que la comunidad de Motozintla de Mendoza ha sido afectada en varias ocasiones por enormes flujos de escombros de magnitudes catastróficas. Datos recientes (Novelo et al., 2013, Sánchez-Núñez et al., 2015), indican que los depósitos en abanicos aluviales contienen el registro de grandes inundaciones y generación de flujos de escombros que provocaron grandes desastres, como los registrados en 1998 y 2005. Se estimó que la recurrencia de grandes eventos de depósito de flujos de escombros está asociado a precipitaciones 

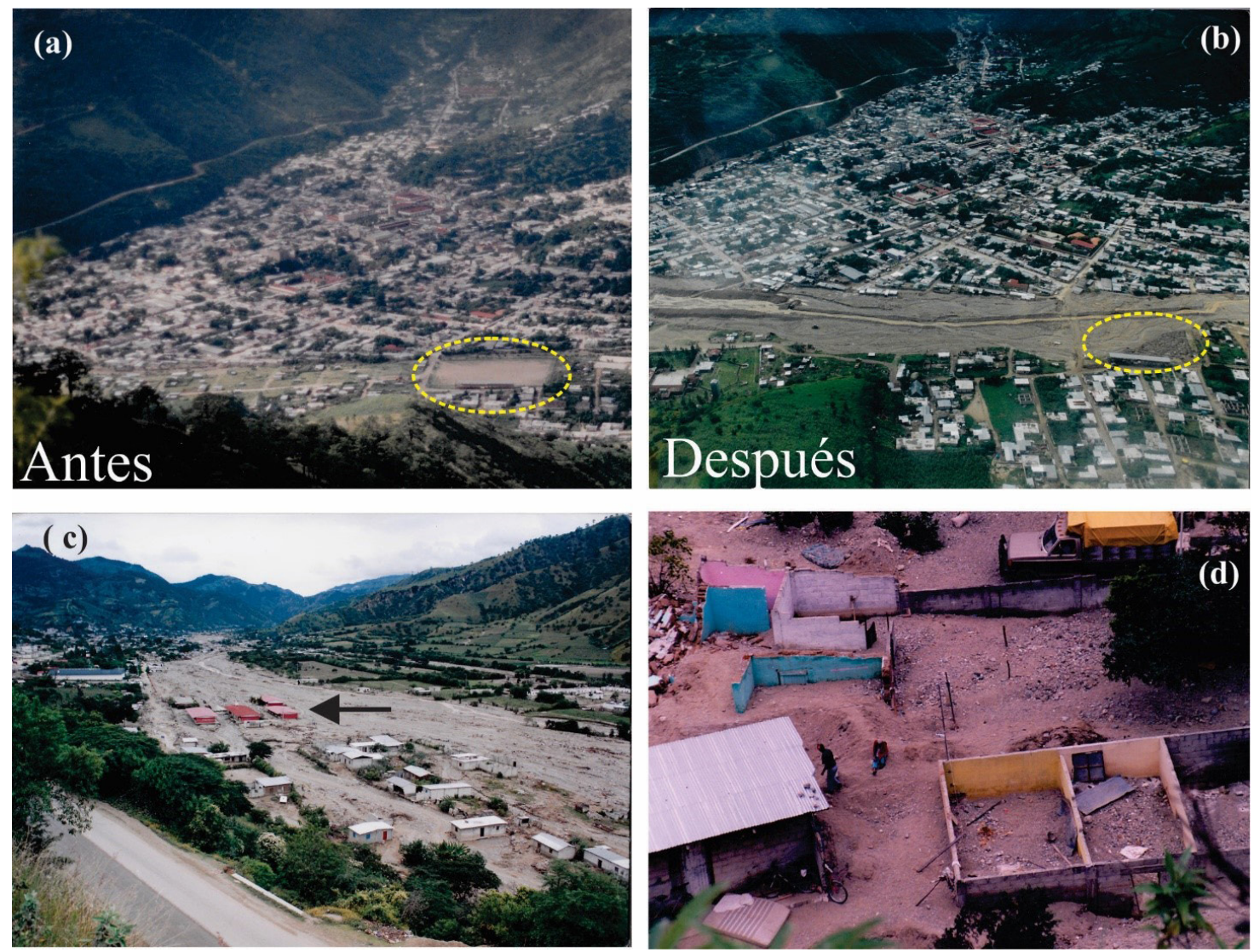

Figura 10 Desastre por inundación y depósito de materiales en la cuenca de Motozintla. (a) "Antes", se muestra una imagen panorámica de la cabecera municipal de Motozintla en el año de 1993. Con círculo punteado aparecen las gradas y el campo de futbol. (b) "Después", (1998) se observa enorme depósito de flujos de escombros (ancho aproximado de $90 \mathrm{~m}$ ), cubriendo por completo el campo de futbol y edificaciones construidas en la ribera y sobre el cauce del río Xelajú Grande. c) vista de E - O del río Xelajú Grande, donde se observa la destrucción de la escuela preparatoria de la cabecera municipal de Motozintla de Mendoza (flecha negra). d) Afectaciones a zonas urbanas, nótese la invasión de los flujos de escombros al interior de las casas. Fotografías cortesía de V.H. Robledo.

pluviales extraordinarias cuya magnitud se repite entre 30 y 40 años (Caballero et al., 2006).

El hecho de que Motozintla esté ubicada en una zona tectónicamente activa (sobre la traza de la falla Polochic) y por tanto sujeta a constante sismicidad, que la ciudad esté asentada en la confluencia de tres grandes corrientes fluviales (Xelajú Grande, Mina, Allende) y que se localice sobre grandes depósitos de flujos de escombros (Figura 4), indica una situación de peligro inminente para la comunidad de Motozintla (Figura 10).

La condición de peligro geológico se magnifica por la presencia de fenómenos hidrometeorológicos anómalos y sobre todo por las actividades antropogénicas que se desarrollan en la región: la excesiva deforestación de bosques, las actividades agrícolas, la ganadería extensiva, las asentamientos humanos irregulares sobre los cauces de ríos y la construcción de caminos en las partes altas de la sierra, son factores que incrementan la inestabilidad de laderas, la cantidad de materiales disponibles para su remoción y la situación de vulnerabilidad de la población.

\section{Conclusiones}

La cuenca de Motozintla se localiza en una zona de montaña, donde los procesos erosivos se han incrementado por la presencia de fenómenos hidro- 
meteorológicos extremos, la dinámica de la falla Polochic y las actividades antrópicas desarrolladas al interior de la cuenca.

Esta cuenca está formada por una gran cantidad de abanicos aluviales, cada uno posee una estratigrafía variable pero con características granulométricas similares entre los depósitos indicando fuentes de origen común. Estos depósitos representan aportes individuales de las microcuencas localizadas perpendicularmente a la planicie.

La distribución granulométrica de cada depósito está asociada a un ambiente de pie de monte con alta energía en las corrientes fluviales. También se puede concluir que la recurrencia de los depósitos puede estar asociada a eventos hidrometeorológicos severos y algunos sísmicos que originan flujos de escombros en forma simultánea en cada microcuenca, formando abanicos aluviales.

La Distribución Granulométrica Total de los depósitos en los abanicos aluviales confirman que, en su mayoría, se trata de flujos de escombros mal seleccionados (1.87) a extremadamente mal seleccionados (4.16); la mayoría de los materiales fueron transportados por tracción y saltación, asociados a corrientes hídricas de alta energía.

En cambio los depósitos de flujos hiperconcentrados $(\mathrm{FH})$ formados a partir de la remobilización de depósitos de flujos piroclásticos, presentan una granulometría más fina $(<4 \phi)$ con una buena selección (0.7). Los depósitos de FH se presentan de forma esporádica en los abanicos aluviales y están constituidos principalmente por el retrabajo del flujo piroclástico Los Chocoyos o por la transformación de los FE.

La recurrencia de los depósitos descritos y los eventos modernos ocurridos en 1998 y 2005 indican que la comunidad de Motozintla está expuesta a un peligro constante por flujos de escombros e inundaciones. Los resultados presentados en este trabajo podrían ser utilizados por investigadores interesados en estudios de la evaluación del riesgo y determinar el nivel potencial de amenaza para la población.

\section{Agradecimientos}

Esta investigación se realizó con apoyo del proyecto IPN-SIP-CIIEMAD 20151929 y 20161470 de J.M. Sánchez-Núñez y el proyecto CONACYT 5624 de D. Novelo.

\section{Referencias}

Allen, J.R., 1970, A quantitative model of grain size and sedimentary structures in lateral deposits: Geological Journal, 7, 129-146.

Anderson, T., Burkart, B., Clemons, R., Bohnenberger, O., Blont, D., 1973, Geology of the Western Altos Cuchumatanes, Northwestern Guatemala: Geological Society of America Bulletin, 84, 805-826.

Ashley, G.M., 1978, Interpretation of polimodal sediments: The Journal of Geology, 86-4, 411-421.

Authemayou, C., Brocard, G., Teyssier, C., Simon-Labric, T., Gutiérrez, A., Chiquín, E.N., Morán, S., 2011, The CaribbeanNorth America-Cocos triple junction and the dynamics of the Polohic-Motagua fault systems: Pull-up and zipper models: Tectonics, 30, TC3010, doi: 10.1029/2010TC002814.

Bahrami, S., 2013, Tectonic controls on the morphometry of alluvial fans around Danehkhoshk anticline, Zargos, Iran: Geomorphology, 180-181, 217-230.

Basu, S., Sarkar, S., 1990, Development of alluvial fans in the foothills of the Darjeeling Himalayas and their geomorphological and pedological characteristics, en Rachocki, A., Church, M. (eds.), Alluvial fans, a field approach: England, John Willey, 321-333.

Boggs, S., 2006, Principles of Sedimentology and Stratigraphy: USA, Pearson Education, Prentice Hall, 662 p.

Briones, F., 2010, Inundados, reubicados y olvidados: Traslado del riesgo de desastres en Motozintla, Chiapas: Revista de Ingeniería, 31, 132-144. 
Burkart, B., 1978, Offset across the Polochic fault of Guatemala and Chiapas, Mexico: Geology, 6, 328-332.

Burkart, B., 1983, Neogene North AmericanCaribbean plate boundary across Northern Central America: offset along the Polochic Fault: Tectonophysics, 99, 251-270.

Caballero, G.A., Macías, J.L., García-Palomo, A., Saucedo, R., Borselli, L., Sarochi, D., Sánchez-Núñez, J.M., 2006, The September 8-9 rain-triggered flows at Motozintla, Chiapas, Mexico: Natural Hazards, 39, 103-126.

Capra, L., Lugo-Hubp, J., Zamorano-Orozco, J.J., 2006, La importancia de la geología en el studio de los procesos de remoción en masa: el caso de Totomoxtla, Sierra Norte de Puebla, México: Boletín de la Sociedad Geológica Mexicana, 58(2), 205-214.

Capra, L., Macias, J.L., 2000, Pleistocene cohesive debris flows at Nevado de Toluca Volcano, central Mexico: Journal of Volcanology and Geothermal Research, 102, 149-168.

Capra, L., Macías, J.L., 2002, The cohesive Naranjo debris-flow deposit $\left(10 \mathrm{~km}^{3}\right)$ : A dam breakout flow derived from the Pleistocene debris-avalanche deposit of Nevado de Colima Volcano (Mexico): Journal of Volcanology and Geothermal Research, 117, 213-235.

Carballido, P.A., 2008, Análisis comparativo de las reubicaciones urbanas por desastre en Motozintla, Chiapas. El caso de las colonias Nuevo Milenio III y Fraccionamiento Vida Mejor III, entre 1998 y 2005: Tesis de Licenciatura, 133p.

Carfantan, J., 1977, La cobijadura de Motozintla. Un paleoarco volcánico en Chiapas: Revista del Instituto de Geología, UNAM, 1-2, 133-137.

Carrera, L., Capra, L., 2008, Estudio granulométrico comparativo de los depósitos epiclásticos en ambientes volcánicos: Revista Mexicana de Ciencias Geológicas, 25(1), 97-114.
Castillo, M., Muñoz-Salinas, E., Arce, J.L., 2015, Evaluación del sistema erosivo fluvial en el volcán Popocatépetl (México) mediante análisis morfométricos: Boletín de la Sociedad Geológica Mexicana, 67(2), 167-183.

CONAGUA, 2007, Comisión Nacional del Agua, Estadísticas del Agua en México, Secretaría de Medio Ambiente y Recursos Naturales (SEMARNAT), México, 259 p.

Damon, P.E., Shafiqullah, M., Clark, K., 1981, Age trends for igneous activity in relation to metallogenesis in the southern Cordillera, en Dickinson, W., Payne, W.D. (eds.), Relations of tectonics to ore deposits in the southern Cordillera: Arizona Geological Society Digest, 14, 137-153.

Davis, J.C., 1970, Information contained in Sediment-Size Analysis: Mathematical Geology, 2(2), 105-112.

De Pedraza, G.J., 1996, Geomorfología, principios, métodos y aplicaciones: Madrid, Editorial Rueda, 413 p.

Drexler, J.W., Rose, W.I., Sparks, R.S., Ledbetter, M.T., 1980, The Los Chocoyos Ash, Guatemala: A mejor stratigraphic marker in Middle America and in three ocean basins: Quaternary Research, 13, 237-345.

Espíndola, V.H., 1996, Sismicidad asociada a la zona de subducción en Chiapas: Ensenada, Baja California, Mexico, Centro de Investigación Científica y de Educación Superior de Ensenada, Informe parcial, 81 p.

Folk, R.L., 1974, Petrology of sedimentary rocks: Austin, Texas, Hemphill Publishing Company, $184 \mathrm{p}$.

Franco, A., Laserre, C., Lyon-Caen, H., Kostoglodov, V., Molina, E., GuzmánSpeziale, M., Monterosso, D., Robles, V., Figueroa, C., Amaya, W., Barrier, E., Chiquin, L., Moran, S., Flores, O., Romero, J., Santiago, J.A., Manea, M., Manea, V.C., 2012, Fault kinematics in northern Central America and coupling along the subduction interface of the Cocos Plate, from GPS data in Chiapas (Mexico), Guatemala and El 
Salvador: Goephysical Journal International, 189, 1223-1236.

Franco-Ramos, O., Vázquez-Selem, L., Zamorano-Orozco, J.J., Villanueva-Díaz, J., 2017, Edad, dinámica geomorfológica y tipología de barrancas en el sector norte del volcán Popocatépetl, México: Boletín de la Sociedad Geológica Mexicana, 69(1), 1-19.

García-Palomo, A., Vásquez, A., Melo, H., 1987, Interpretación tectónica estructural del valle de Uzpanapa, Veracruz. México: Escuela Superior de Ingeniería y Arquitectura, IPN, Tesis de licenciatura, $83 \mathrm{p}$.

Godínez, A., 2009, Paleomagnetismo de la Formación Todos Santos, Chiapas, México: México, Universidad Nacional Autónoma de México, Centro de Geociencias, Campus Juriquilla, tesis de maestría, $161 \mathrm{p}$.

Gutiérrez-Elorza, M., 2008, Geomorfología: Madrid, España, Pearson Prentice Hall, 898 p.

Guzmán-Speziale, M., 2010, Beyond the Motagua and Polochic faults: Active strike-slip faulting along the Western North America-Caribbean plate boundary zone: Tectonophysics, 426, $17-27$.

Guzmán-Speziale, M., Meneses-Rocha, J., 2000, The North America-Caribbean plate Boundary west of the Motagua Polochic fault system: A fault jog in Southeastern Mexico: Journal of South America Earth Sciences, 13, 459-468.

Harvey, A.M., 2007, Geomorphic instability and change. Introduction: Implication of temporal and spatial scales: Geomorphology, 48(3-4), 153-158.

Inman, D.L., 1952, Measures for describing the size distribution of sediments: Journal of Sedimentary Petrology, 22(3), 125-145.

Kellerhals, R., Bray, I.D., 1971, Sampling procedures for coarse fluvial sediments: Journal of Hydraulics Division, 97(8), 1165-1180.
Luzón, A.A., 1999, Zonación y principales características de los abanicos terciarios del borde norte de la cuenca Ebro, Provincia de Huasca, España: GeoGaceta, 26, 55-58.

Lyon-Caen, H. Barrier, E., Lasserre, C., Franco, A., Arzu, I., Chiquin, L., Chiquin, M., Duquesnoy, T., Flores, O., Galicia, O., Luna, J., Molina, E., Porras, O., Requena, J., Robles, V., Romero, J., Wolf, R., 2006, Kinematics of the North America-Caribbean-Cocos plates in Central America from new GPS measurements across the Polochic-Motagua fault system: Geophysical Research Letters, 33, (19), doi: 10.1029/2006GL027696.

Macías, J.L., García-Palomo, A., Arce, J.L., Siebe, C., Espíndola, J.M., Komorowski, J.C., Scott, K., 1997, Late Pleistocene-Holocene cataclysmic eruptions at Nevado de Toluca and Jocotitlán volcanoes, Central México, en Link, P.K., Kowallis, B.J. (eds.), Proterozoic to Recent Stratigraphy, Tectonics, and Volcanology, Utah, Nevada, Southern Idaho, and central Mexico: BrighamYoung University, Geology Studies, 42(I), 493-528.

Macías, J.L., Capra, L., Scott, K., Espíndola, J.M., García-Palomo, A., Costa, J.E., 2004, The 26 May 1982 breakout flows derived from failure of a volcanic dam at El Chichón, Chiapas, Mexico: Geological Society of America Bulletin, 116(1-2), 233-246.

Macías, J.L., Arce, J.L., García-Palomo. A., Mora, J.C., Layer, P.W., Espíndola, J.M., 2010, Late-Pleistocene flank collapse triggered by dome growth at Tacaná Volcano, MéxicoGuatemala, and its relationship to the regional stress regime: Bulletin of Volcanology, 72(1), 33-53.

McManus, J., 1988, Grain size determination and interpretation, en Tucker, M., 1988 (ed.), Techniques in sedimentology: Oxford, Blackwell Scientific Publication, 394 p.

Mérida, R., 1976, Principios generales de los cobres porfídicos y su relación con el 
depósito cuprífero de Tolimán, Municipio de Motozintla, Chiapas: Ciudad de México, Instituto Politécnico Nacional, Escuela Superior de Ingeniería y Arquitectura, Tesis de licenciatura, $92 \mathrm{p}$.

Miall, A.D., 2000, Principles of Sedimentary Basin Analysis: New York, Springer, 616 p.

Miall, A.D., 2006, The Geology of Fluvial Deposits, Sedimentary Facies, Basin Analysis, and Petroleum Geology: New York, Springer, $582 \mathrm{p}$.

Molina-Garza, R., Geissman, J., Wawrzyniec, T., Peña Alonso, T., Iriondo, A., Weber, B., Aranda-Gómez, J., 2015, Geology of the coastal Chiapas (Mexico) Miocene plutons and the Tonalá shear zone: Syntectonic emplacement and rapid exhumation during sinistral transpression: Lithosphere, Geological Society of America, 7(3), 257-274.

Moravec, D., 1983, Study of the Concordia Fault System, Near Jerico, Chiapas, México: E.U.A., The University of Texas at Arlington, Tesis de maestría, $148 \mathrm{p}$.

Moreno, S., 1977, Estudio geológico regional del área de Acatepagua, Motozintla, Chiapas: México, Instituto Politécnico Nacional, Escuela Superior de Ingeniería y Arquitectura, Tesis de licenciatura, 51 p.

Muehlberger, W., Ritchie, A., 1975, CaribbeanAmericas plate boundary in Guatemala and southern Mexico as seen on Skylab IV orbital photography: Geology, 3, 232-235.

Múgica, R., 1987, Estudio petrogenético de las rocas ígneas y metamórficas del Macizo de Chiapas, México: Instituto Mexicano del Petróleo, Reporte C-2009, 96 p.

Mulder, T., Alexander, J., 2001, The physical character of subaqueous sedimentary density flows and their deposits: Sedimentology, 48, 269-299.

Murcia, H., Macías, J.L., 2009, Registro geológico de inundaciones recurrentes e inundación del 4 de octubre de 2005 en la ciudad de Tapachula, Chiapas, México: Revista Mexicana de Ciencias Geológicas, 26, 1-17.

Neves, M.A., Morales, N., Saad, A.R., 2005, Facies analysis of tertiary alluvial fan deposits in the Jundiaí region, Sao Paulo, southeastern Brazil: Journal of South American Earth Sciences, 19, 513-524.

Nichols, G., 2009, Sedimentology and stratigraphy: UK, John Wiley, 419 p.

Novelo, D., Oropeza, O., Macías, J.L., Alcántara, I., Mansilla, E., Ramos, S., 2013, Atlas de factores de riesgos de la cuenca de Motozintla, Chiapas: México, Conacyt-UNAM, 222 p.

Peraldo, G., Montero, W., 1999, Sismología histórica de América Central, Giudad de México, Instituto Panamericano de Geografía e Historia, México, D.F., México, 53, $347 \mathrm{p}$.

Rabek, K., Ledbetter, M.T., Williams, D.F, 1985. Tephrochronology of western Gulf of Mexico for the last 185000 years: Quaternary Research, 23, 403-416.

Rosiwal, A., 1898, Über geometrische Gesteinsanalysen; ein einfacher Weg zur ziffermässigen Festellung des Quantitätsverhältnisses der Mineralbestandtheile gemengter Gesteine: Verhandlungen der Kaiserlich-Koeniglichen Geologischen Reichsanstalt, Wien, 5/6, 143-175.

Salazar,J., 2008, Geología, petrología y geoquímica del Macizo de Chiapas, área de Motozintla de Mendoza: México, Universidad Nacional Autónoma de México, Facultad de Ingeniería, tesis de licenciatura, $68 \mathrm{p}$.

Sánchez-Núñez, J.M., Macías, J.L., Zamorano, J.J., Saucedo, R., Torres-Hernández, J.R., Novelo, D., 2012, Mass movement processes at Motozintla Basin, Southern Mexico: Geofisica Internacional, 51(2), 169-184.

Sánchez-Núñez, J.M., Macías, J.L., Saucedo, R., Zamorano, J.J., Novelo, D., Mendoza, M.E., Torres-Hernández, J.R., 2015, Geomorphology, internal structure and 
evolution of alluvial fans at Motozintla, Chiapas, México: Geomorphology, 230, $1-12$.

Sarocchi, D., Borselli, L., Macías, J.L., 2005, Construcción de perfiles granulométricos de depósitos piroclásticos por métodos ópticos: Revista Mexicana de Ciencias Geológicas, 22(3), 371-382.

Saucedo, R., Macías, J.L., Bursik, M., 2004, Pyroclastic flow deposits of the 1991 eruption of Volcán de Colima, Mexico: Bulletin of Volcanology, 66, 291-306.

Saucedo, R., Macías, J.L., Sarocchi, D., Bursik, M., Rubb, B., 2008, The rain-triggered Atenquique volcaniclastic debris flow of October 16, 1955 at Nevado de Colima Volcano, Mexico: Journal of Volcanology and Geothermal Research, 173, 69-83.

Schaaf, P., Weber, B., Weis, P., Gross, A., OrtegaGutiérrez, F., Köhler, H., 2002, The Chiapas Massif (Mexico) revised: New geologic and isotopic data for basement characteristics, en Miller, H., ed., Contributions to Latin American Geology: Neues Jahrbuch für Geologie und Paläontologie Abhandlung, 225, 1-23.

SEDESOL, 2017, Secretaría de Desarrollo Social, Unidad de Microrregiones, Cédulas de Información Municipal (SCIM), Municipios (en línea): ZAP Rural, disponible en: <http://www. microrregiones.gob.mx/zap/datGenerales. aspx?entra $=$ zap\&ent $=07 \&$ mun $=057>$, consultado 13 marzo de 2017.

Visher, G., 1969, Grain size distributions and depositional processes: Journal of Sedimentary Petrology, 39(3), 1074-1 106.

Walker, J.A., Templeton, S., Cameron, B.I., 2006, The chemistry of spring waters and fumarolic gases encircling Santa Maria Volcano, Guatemala: Insights into regional hydrothermal activity and implications for volcano monitoring, en Rose, W.L., Bluth, G.J.S., Carr, M.J., Ewert, J.W., Patino, L.C., Vallance, J.W. (eds.), Volcanic Hazards in Central America: Geological Society of America Special Paper 412, 59-83.

Weber, B., Gruner, B., Hecht, L., Molina-Garza, R., Köhler, H., 2002, El descubrimiento de basamento metasedimentario en el Macizo de Chiapas: La "Unidad la Sepultura": GEOS, 22(1), 2-11.

Weber, B., Cameron, K., Osorio, M., Schaaf, P., 2005, A Late Permian Tectonothermal Event in Grenville Crust of the Southern Maya Terrane: U-Pb Zircon Ages from the Chiapas Massif, Southeastern Mexico: International Geology Review, 47, 509-529.

Weber, B., Valencia, V., Schaaf, P., Pompa-Mera, V., Ruiz, J., 2008, Significance of Provenance Ages from the Chiapas Massif Complex (Southeastern Mexico): Redefining the Paleozoic Basement of the Maya Block and its Evolution in a Peri-Gondwanan Realm: Journal of Geology, 116, 619-639.

White, R., 1985, The Guatemala earthquake of 1816 on the Chixoy-Polochic fault: Bulletin of the Seismological Society of America, 75, 455-473.

Williams, S., Self, S., 1983, The october 1902 plinian eruption of Santa María volcano, Guatemala: Journal of Volcanology and Geothermal Research, 16, 36-56.

Wohletz, K., Sheridan, M.F., Brown, W.K., 1989, Particle size distribution and the sequential fragmentation/transport theory apply to volcanic ash: Journal of Geophysical Research, 94, 15703-15721.

Zhou, G.D., Gui, P., Tang, J.B., Chen, H.Y., Zou, Q., Sun, Q.C., 2015, Experimental study on the triggering mechanisms and kinematic properties of large debris flows in Wenjia Gully: Engineering Geology, 194, 52-61. 


\section{Anexo 1}

Se muestra información complementaria de los histogramas generados para la caracterización granulométrica de los depósitos de abanicos aluviales en la cuenca de Motozintla, Chiapas, México.

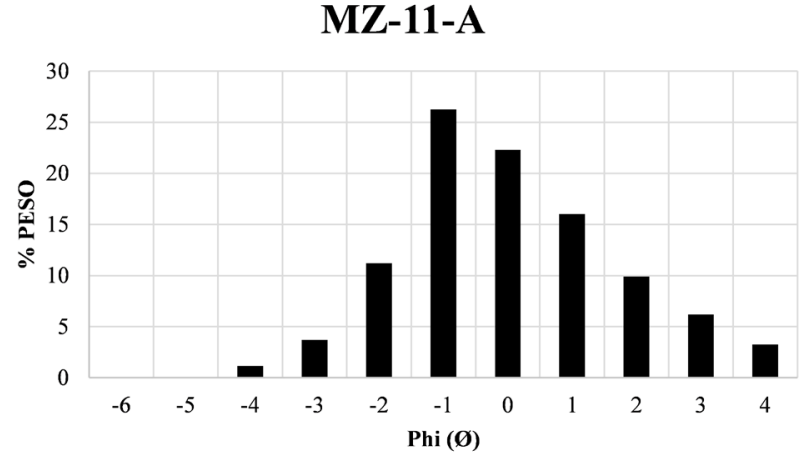

MZ-11-E

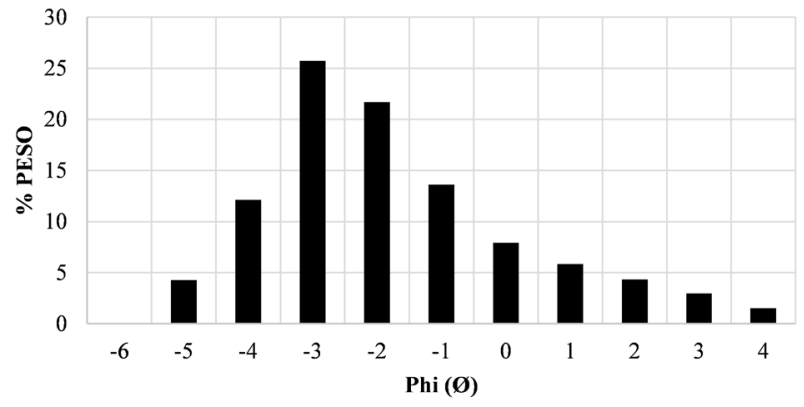

MZ-14
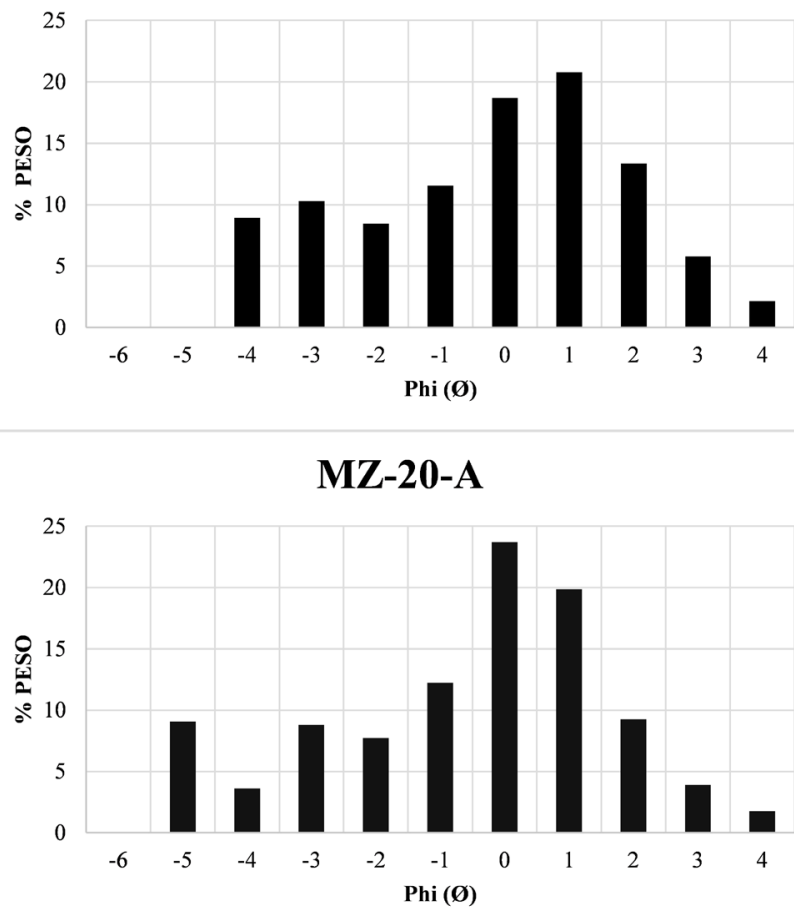

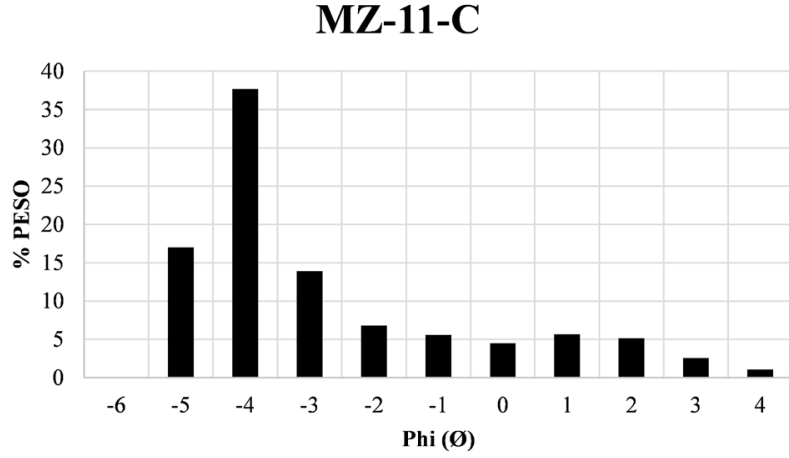

MZ-12

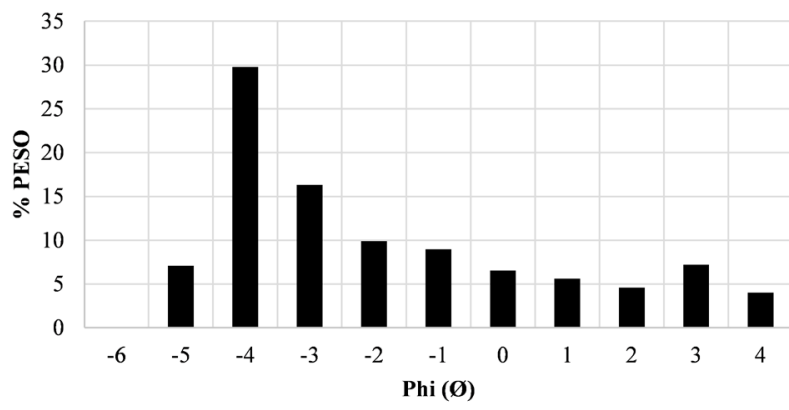

MZ-16

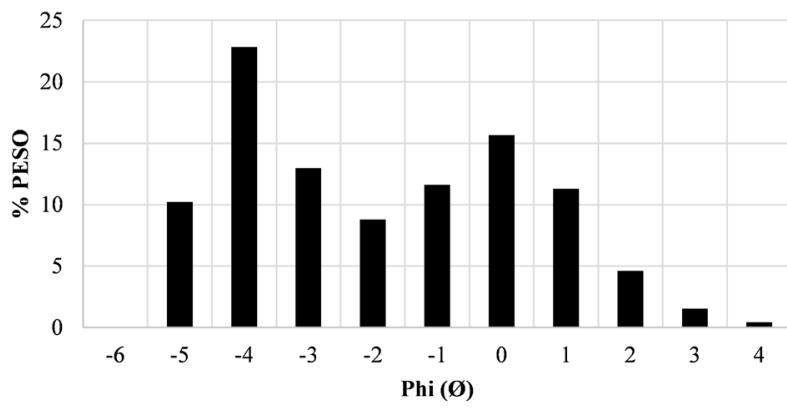

MZ-20-C

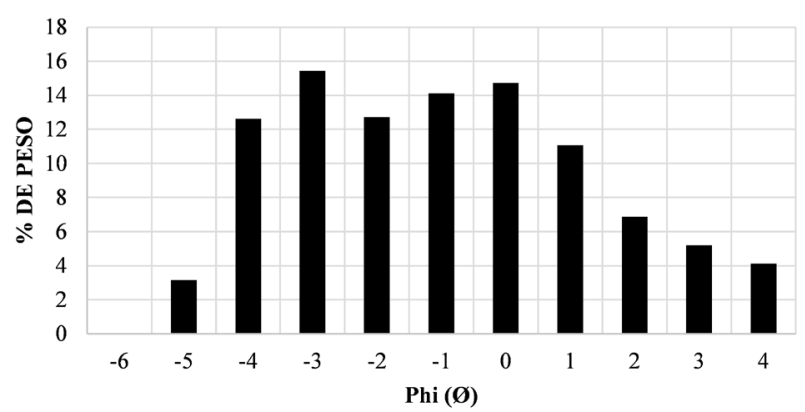



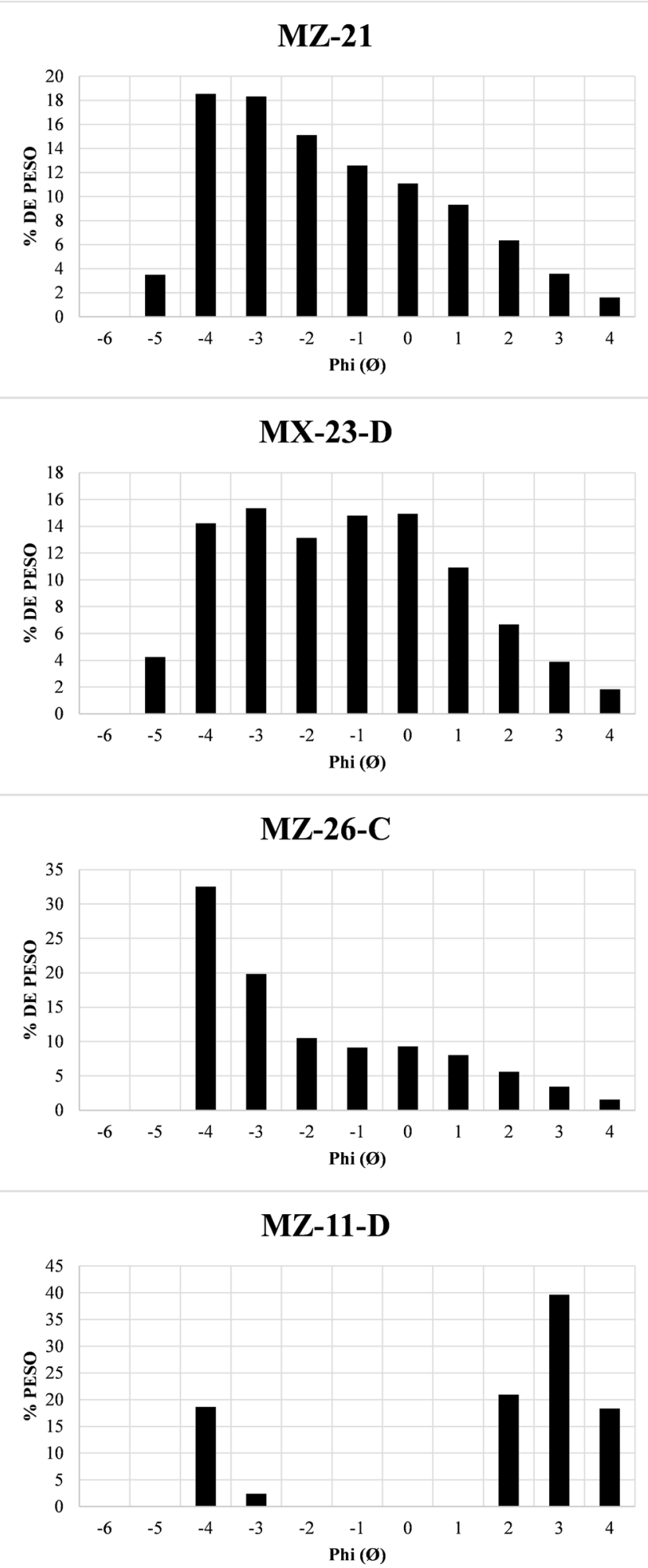
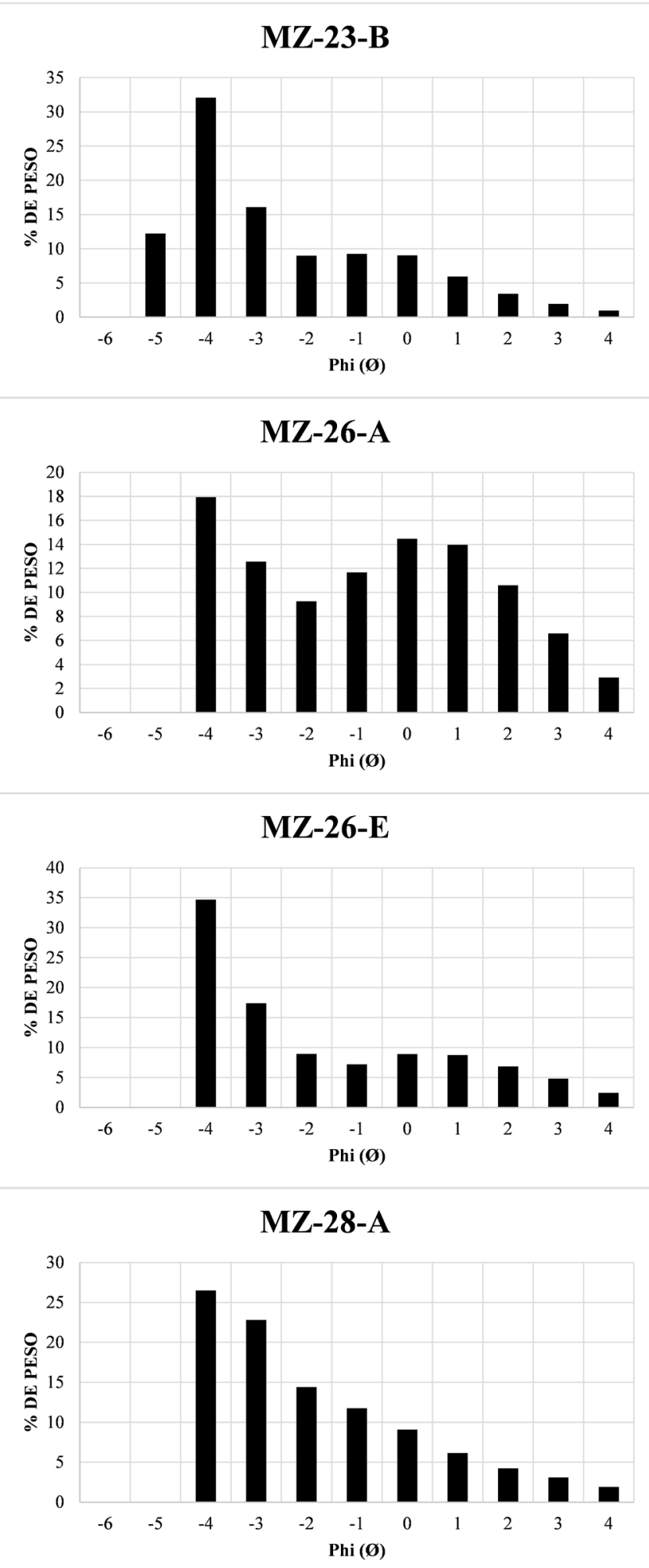

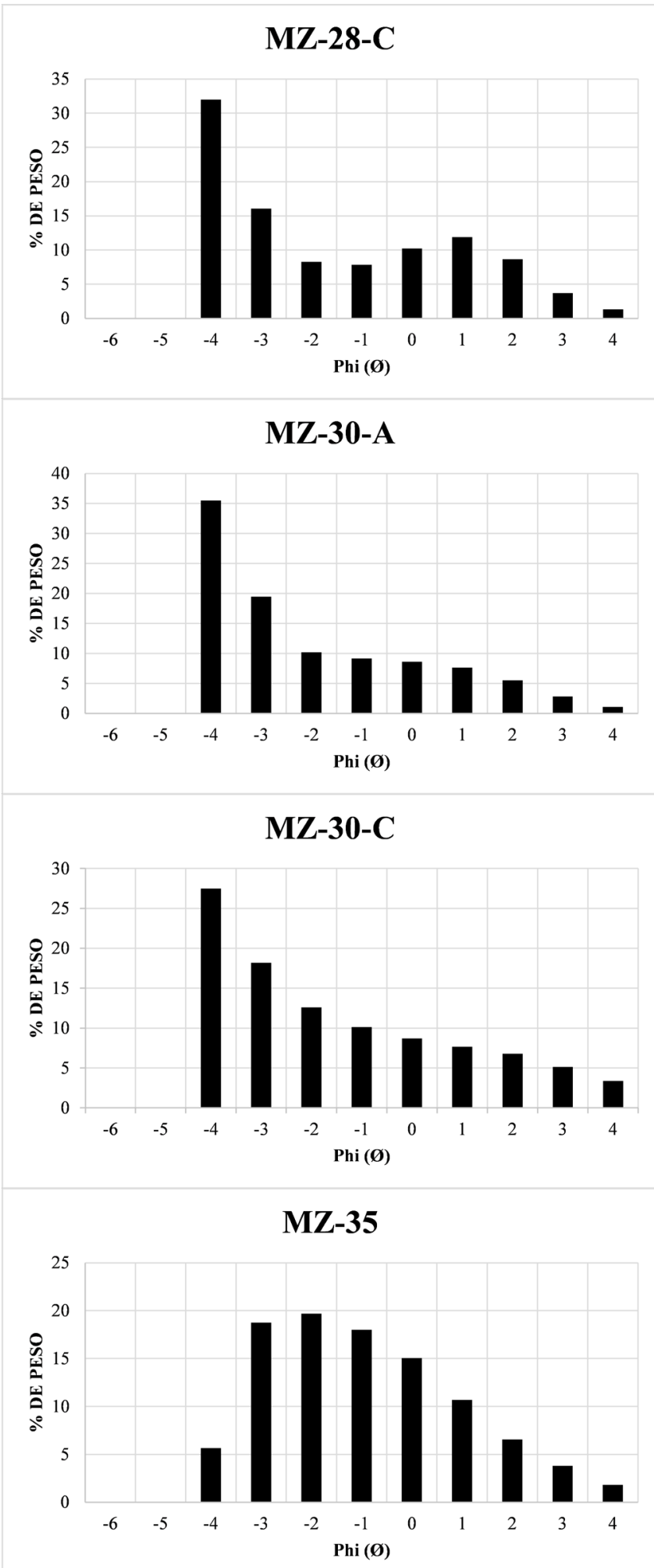
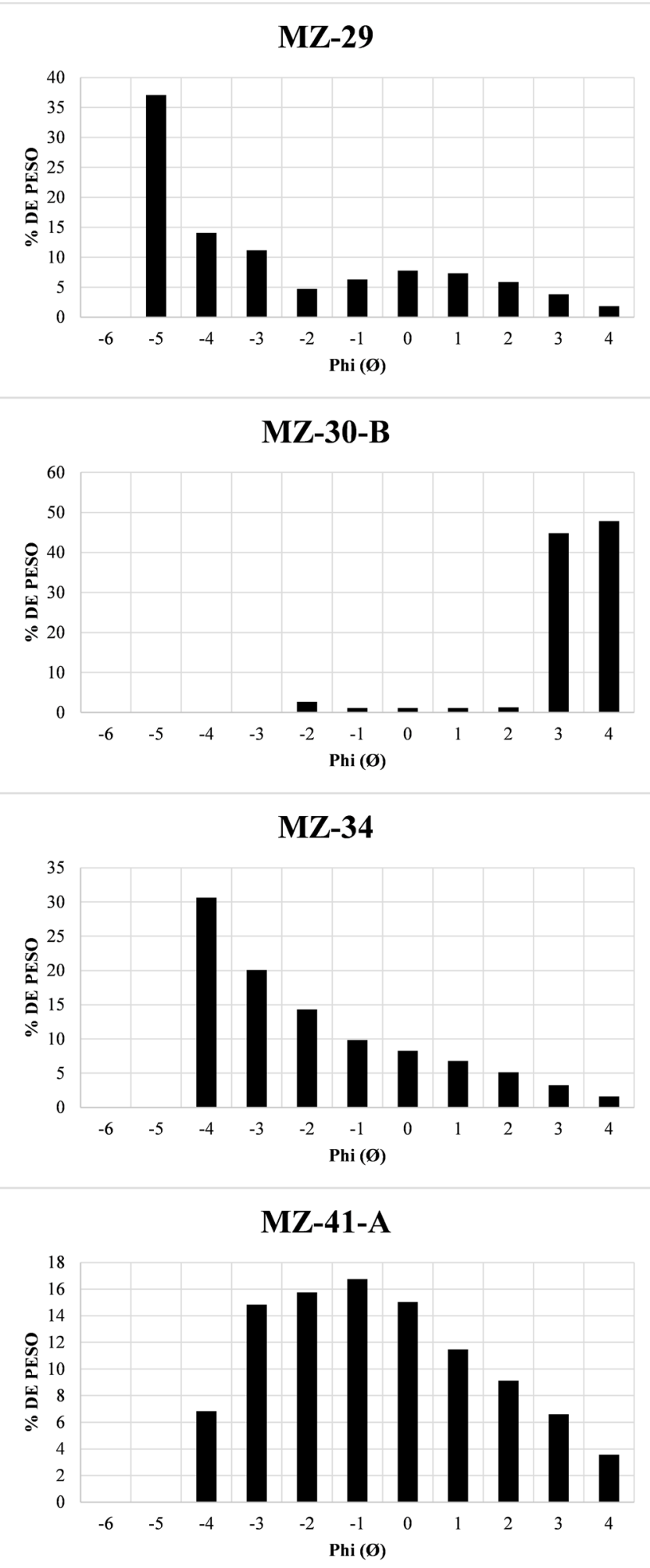

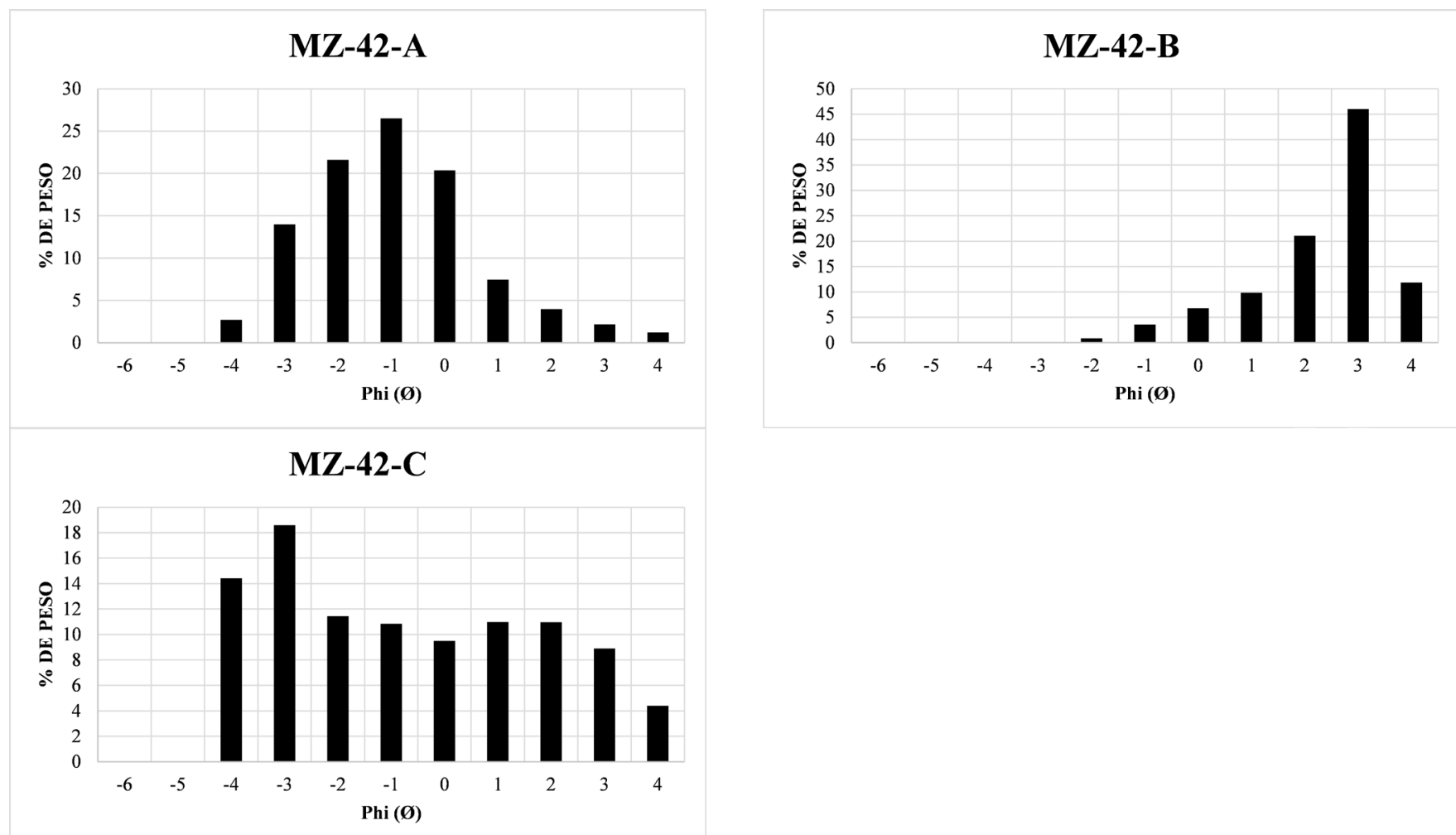